\title{
Identification of a neuronal population in the telencephalon essential for fear conditioning in zebrafish
}

Pradeep Lal ${ }^{1,2,3}$, Hideyuki Tanabe ${ }^{1}$, Maximiliano L. Suster ${ }^{1,4}$, Deepak Ailani', Yuri Kotani ${ }^{1}$, Akira Muto ${ }^{1}$, Mari Itoh ${ }^{1}$, Miki Iwasaki ${ }^{1,5}$, Hironori Wada ${ }^{1,5}$, Emre Yaksi $^{3}$ and Koichi Kawakami ${ }^{1,2^{*}}$ (i)

\begin{abstract}
Background: Fear conditioning is a form of learning essential for animal survival and used as a behavioral paradigm to study the mechanisms of learning and memory. In mammals, the amygdala plays a crucial role in fear conditioning. In teleost, the medial zone of the dorsal telencephalon ( $\mathrm{Dm}$ ) has been postulated to be a homolog of the mammalian amygdala by anatomical and ablation studies, showing a role in conditioned avoidance response. However, the neuronal populations required for a conditioned avoidance response via the Dm have not been functionally or genetically defined.
\end{abstract}

Results: We aimed to identify the neuronal population essential for fear conditioning through a genetic approach in zebrafish. First, we performed large-scale gene trap and enhancer trap screens, and created transgenic fish lines that expressed Gal4FF, an engineered version of the Gal4 transcription activator, in specific regions in the brain. We then crossed these Gal4FF-expressing fish with the effector line carrying the botulinum neurotoxin gene downstream of the Gal4 binding sequence UAS, and analyzed the double transgenic fish for active avoidance fear conditioning. We identified 16 transgenic lines with Gal4FF expression in various brain areas showing reduced performance in avoidance responses. Two of them had Gal4 expression in populations of neurons located in subregions of the Dm, which we named 120A-Dm neurons. Inhibition of the 120A-Dm neurons also caused reduced performance in Pavlovian fear conditioning. The 120A-Dm neurons were mostly glutamatergic and had projections to other brain regions, including the hypothalamus and ventral telencephalon.

Conclusions: Herein, we identified a subpopulation of neurons in the zebrafish Dm essential for fear conditioning. We propose that these are functional equivalents of neurons in the mammalian pallial amygdala, mediating the conditioned stimulus-unconditioned stimulus association. Thus, the study establishes a basis for understanding the evolutionary conservation and diversification of functional neural circuits mediating fear conditioning in vertebrates.

Keywords: gene trapping, enhancer trapping, transposable element, fear conditioning, Pavlovian conditioning, botulinum neurotoxin, Gal4-UAS, dorsomedial telencephalon, amygdala

\footnotetext{
* Correspondence: kokawaka@nig.ac.jp

'Division of Molecular and Developmental Biology, National Institute of

Genetics, Mishima, Shizuoka 411-8540, Japan

2Department of Genetics, SOKENDAl (The Graduate University for Advanced

Studies), Mishima, Shizuoka 411-8540, Japan

Full list of author information is available at the end of the article
} 


\section{Background}

Fear conditioning is a type of learning through which animals learn to predict an aversive event from a correlated environmental cue. In mammals, the amygdala plays essential roles in this type of learning [1,2]. The mammalian amygdala is a complex and anatomically heterogeneous structure, consisting of approximately 20 subnuclei that are derivatives of the pallial and subpallial portions of the telencephalon. The pallial amygdala consists of cortical nuclei and basolateral nuclei (BLA), containing predominantly glutamatergic neurons, whereas the subpallial amygdala consists of medial and central nuclei (CeA), containing predominantly GABAergic neurons [3-5]. The roles of these nuclei in fear conditioning have been studied extensively by producing nucleispecific lesions. The BLA has been shown to serve as the sensory interface essential for the association of a conditioned (CS) and an unconditioned stimulus (US). The CeA then receives inputs from BLA through intraamygdaloid circuitry and serves as the primary output structure, with projections to other brain regions and controlling fear responses [1, 2, 6, 7].

Fear conditioning is an evolutionarily conserved behavior, and both active avoidance and Pavlovian fear conditioning have been described in teleost fish [8-11]. In teleost, it has been hypothesized that the forebrain is formed by a mechanism called eversion, while the mammalian forebrain is formed by evagination. Additionally, the teleost telencephalon is composed of area dorsalis and area ventralis, which are homologous to the pallium and subpallium and are rich in glutamatergic and GABAergic neurons, respectively [12-15]. From neuroanatomical and hodological studies, it has been proposed that the medial zone of the dorsal telencephalon (Dm) is a homolog of the mammalian amygdala $[16,17]$. A functional study on the telencephalic region important for fear conditioning was performed by ablation experiments [18], wherein goldfish were trained in active avoidance fear conditioning and, after acquisition of the conditioned avoidance response, the medial pallium (MP), including the Dm area, was ablated by surgery. The MP-lesioned fish exhibited a deficit in performing the avoidance response, indicating that the MP area was essential for retention of the conditioned avoidance response. Although the functional study supported the hypothesis that the teleost Dm is a homolog of the mammalian amygdala, the lesions created were quite large, and specific neuronal populations or circuits essential for fear conditioning have yet to be explored.

In zebrafish, Lau et al. [19] analyzed c-fos expression patterns in the brain when fish performed a light/dark choice (light-avoidance) behavior, and found that $c$-fos expression was detected in cells in Dm and other brain regions, including the hypothalamus and ventral telencephalon. von Trotha et al. [20] also analyzed cfos expression in the zebrafish brain during administration of amphetamine, a drug of abuse, and an amphetamine-induced place preference behavior, and detected $c$-fos expression in the Dm area. Thus, these studies suggest the involvement of neurons in Dm in emotional and motivational behaviors. However, the cells found in these studies are not genetically labeled, and are therefore not manipulatable, and the requirements for behaviors are unknown.

Herein, we aim to identify the neuronal population essential for fear conditioning through a genetic approach in zebrafish. In previous studies [21, 22], we developed transposon-mediated gene trap and enhancer trap methods, and generated transgenic fish lines that expressed Gal4FF, an engineered Gal4 transcription activator, in specific organs, tissues and cells, including specific neuronal populations. Further, we demonstrated that, by taking advantage of the Gal4-UAS binary system, the activity of such specific neurons can be inhibited by targeted expression of the tetanus neurotoxin gene $[21,23]$. In this study, we applied this powerful approach to explore the adult brain function. First, we performed large-scale gene trap and enhancer trap screens and identified transgenic fish lines that expressed Gal4FF in various different regions in the adult brain. Second, we selected lines expressing Gal4FF predominantly in the forebrain, crossed them with the UAS-botulinum neurotoxin fish that contained a modified botulinum toxin (BoTx) gene downstream of UAS [24], and analyzed behaviors of the double transgenic fish by using fear conditioning paradigms. Finally, we found transgenic fish lines expressing Gal4FF in a subpopulation of neurons in Dm that showed reduced performance in fear conditioning when crossed with the UAS:BoTx fish. Thus, the present study genetically identified the neuronal population in zebrafish essential for fear conditioning, which may be a functional equivalent of the mammalian amygdala.

\section{Results}

Identification of transgenic fish with Gal4FF expression in the adult brain

The outline of this study is shown in Fig. 1a. We performed large-scale genetic screens by using Tol2 transposon-based gene trap and enhancer trap constructs [21], and generated transgenic fish that expressed Gal4FF, an engineered Gal4 transcription activator, in spatially and temporally restricted fashions at the embryonic stages. From this collection, we selected 349 transgenic fish, including 174 lines with Gal4FF expression in the central nervous system (CNS) and 175 lines without CNS expression at the embryonic stages, crossed them with the UAS:GFP fish, and analyzed the GFP expression 


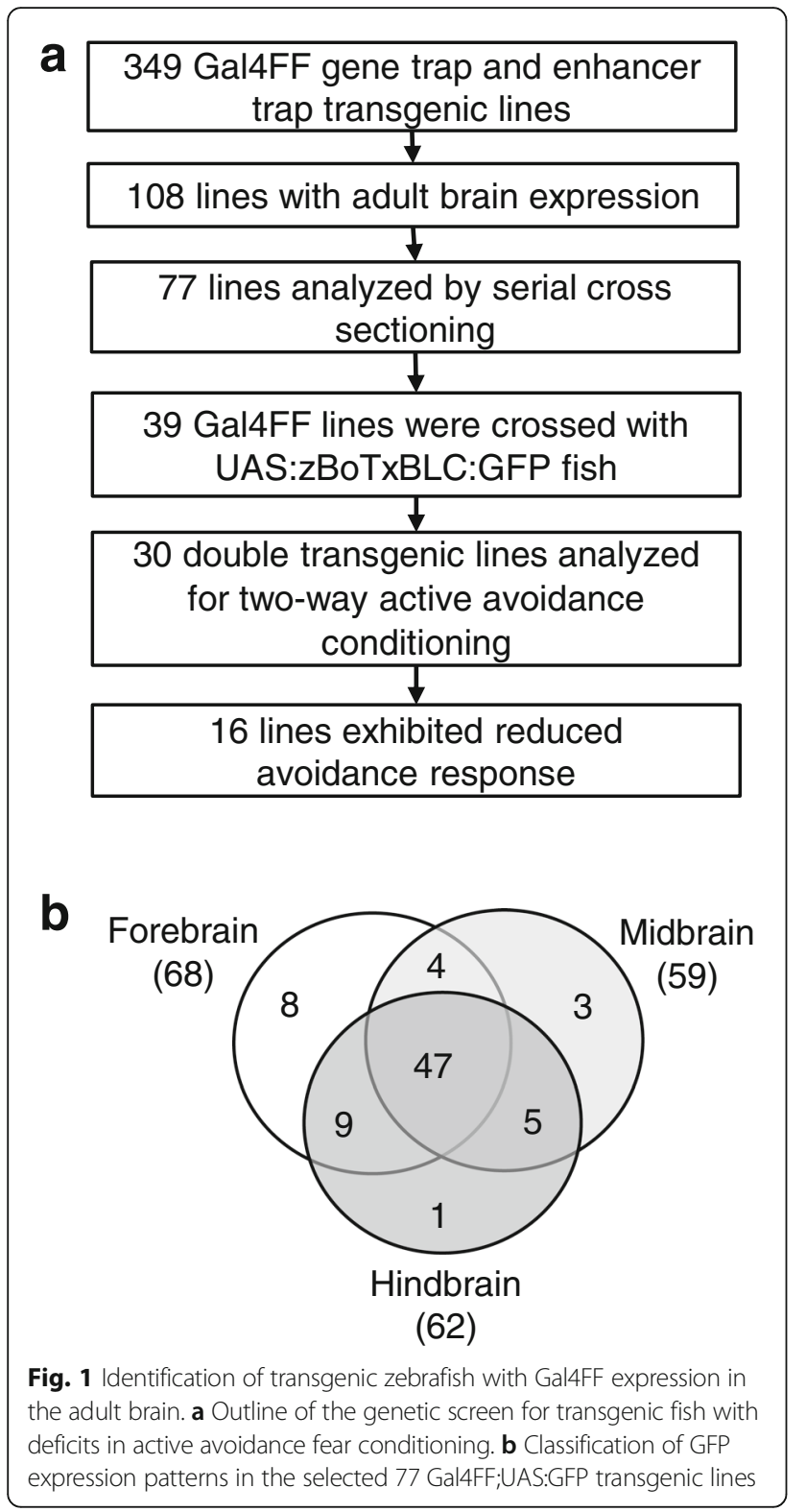

patterns at the adult stage. We first observed their brains externally, then opened the skulls, and identified 108 lines with detectable levels of GFP expression in the adult brain. Among those, 93 already had Gal4FF expression in the CNS at embryonic stages (93/174; 53\%), whereas 15 did not $(15 / 175 ; 8.6 \%)$. Thus, 93 lines with adult brain expression patterns would have been obtained by screening 174 embryonic CNS lines, suggesting prescreening for CNS expression at embryonic stages should enrich fish with Gal4FF expression in the adult brain. From these 108 lines, we selected 77 lines that showed strong GFP expression, and analyzed them further by making coronal sections. The GFP (Gal4FF) expression was observed in the forebrain (68 lines), midbrain (59 lines), and hindbrain (62 lines) (Fig. 1b).
Identification of Gal4FF transgenic fish with deficits in two-way active avoidance fear conditioning

We aimed to identify neuronal populations essential for fear conditioning. For this purpose, we set up an assay system for two-way active avoidance conditioning using a shuttle box with two compartments (Fig. 2a). Green LEDs were used as a CS, and electric shocks given $10 \mathrm{~s}$ after CS were used as a US. When fish moved to another compartment prior to the US, which was defined as "escape" or "active avoidance", the shock was not given. Ten trials per day were conducted on 5 consecutive days (Fig. 2b). In this paradigm, wild type zebrafish showed increased escape responses through the 5 days, compared with fish to which only CS was given (Fig. 2c-e). Thus, active avoidance conditioning using this protocol was efficient and reproducible (Additional file 1: Movie S1).

In order to inhibit neuronal functions, we constructed a transgenic fish line that carried a codon-optimized botulinum toxin $B$ light chain gene fused to the EGFP sequence downstream of the UAS sequence (UAS:zBoTxBLC:GFP). We have already shown that the UAS:zBoTxBLC:GFP line could efficiently inhibit neuronal activities in combination with Gal4 drivers at the larval stages [24]. From the 77 "adult brain" Gal4FF lines, we selected 39 lines that had rather restricted Gal4 expression in the forebrain and crossed them with the UAS:zBoTxBLC:GFP line. While nine double transgenic lines exhibited lethal locomotion deficits during larval stages, 30 double transgenic lines could survive to adulthood. We then analyzed the 30 Gal4FF;UAS:zBoTxBLC:GFP double transgenic fish by using the two-way active avoidance paradigm; 14 lines performed the avoidance response comparable to wild type fish and 16 lines showed significantly reduced performance in acquisition of the avoidance response (Fig. 3a-f, Additional file 2: Figure S1). We analyzed all of these 16 lines by Southern blot hybridization, and confirmed that the Gal4FF expression patterns were generated by single insertions of either the gene or enhancer trap construct.

We then analyzed the 16 Gal4FF;UAS:GFP lines by making serial cross sections (Fig. 4, Additional file 3: Figure S2); 14 lines (hspGGFF10C, hspGGFF20A, hspGFF55B, SAGFF36B, SAGFF70A, SAGFF81B, SAGFF120A, SAGFF226F, SAGFF228A, SAGFF231A, SAGFF233A, SAGFF234A, SAGFF234D, and hspGFFDMC12A) showed GFP expression in the telencephalon and other brain regions. In the telencephalon, GFP expression was prominently observed in the medial zone of the dorsal telencephalon (Dm; 6 lines) and in the ventral nucleus of the ventral telencephalon ( $\mathrm{Vv}: 8$ lines). The other two lines, hspGFF38B and hspGFFDMC56B, showed strong GFP expression in the diencephalon and mesencephalon, including the habenula $(\mathrm{Hb})$ nuclei and tegmentum, and in the preoptic area in the diencephalon, respectively. 
a

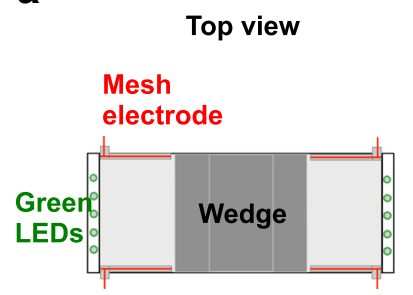

c

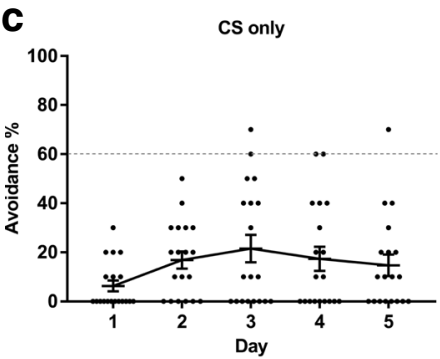

Side view

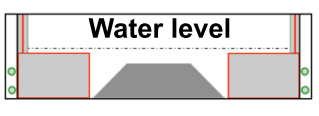

b Habituation Training

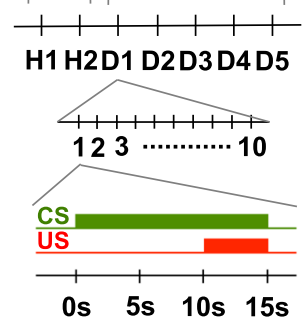

d

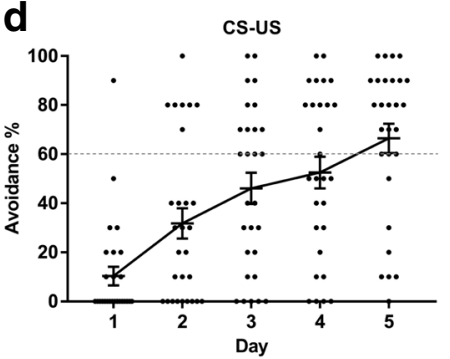

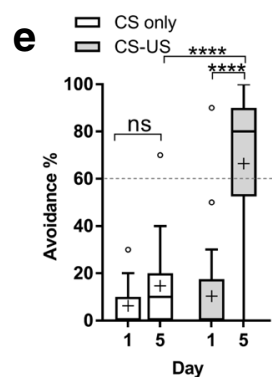

Fig. 2 Two-way active avoidance fear conditioning. a Shuttle box used for active avoidance conditioning. Top view (left) and side view (right). b Scheme for active avoidance conditioning. After habituation for 2 days, fish underwent 10 trials (with $25 \pm 5 \mathrm{~s}$ intervals) per day over 5 consecutive days. In each trial, conditioned stimulus (CS; green LED) was presented followed by an unconditioned stimulus (US; electric shock) $10 \mathrm{~s}$ after CS, for $5 \mathrm{~s}$; after this, both CS and US were turned off. If fish escaped during CS, then US was not given. $\mathbf{c}$, $\mathbf{d}$ Performance of active avoidance response (\%) of wild type fish treated only by CS (CS only: $n=19)(\mathbf{c})$ and by CS and US (CS-US: $n=28)(\mathbf{d})$. e Comparison of performance of active avoidance response of fish treated with CS only and with CS-US in Tukey box plot. Outliers are shown in open circles. Mean is marked by ' + '. Two-way ANOVA, fish groups (CS-US wild type, CS-only wild type, and all double transgenic fish including fish described in Fig. 3 and Additional file 2: Figure S1) $\times$ training days (day 1, day 5), was performed $(F=7.236, P<0.0001)$. Dunnett's multiple comparison post-hoc tests were performed between CS-US and CS-only wild type fish on sessions on day 1 and day 5 (see also Fig. 3 and Additional file 2: Figure S1). The Student's $t$ test was performed on CS-US wild type fish between day 1 and day 5 . ${ }^{* * *} P<0.0001$; ns, not significant

The emx3 enhancer trap lines showed deficits in two-way active avoidance fear conditioning when crossed with the UAS-neurotoxin line

Six lines (SAGFF36B, SAGFF70A, SAGFF120A, SAGFF228A, SAGFF231A, and SAGFF234D) exhibited reduced active avoidance responses and showed Gal4FF expression in the Dm area, which has been postulated to be a homolog of the mammalian amygdala (Fig. $3 g-n$ ). We analyzed these Dm lines by inverse PCR, and determined the integration sites of the gene trap or enhancer trap construct (Table 1).

Among these, two lines, SAGFF70A and SAGFF120A, had rather specific Gal4FF (UAS:GFP) expression patterns in the Dm area (Fig. 3j, k). In the SAGFF70A and SAGFF120A fish, the transposon insertions were located on chromosome 14, both near the $e m x 3$ gene (Fig. 5a). The emx3 gene is expressed in the dorsal telencephalon and part of the diencephalon at larval stages, and in the $\mathrm{Dm}$ area at the adult stage [25-27]. We performed in situ hybridization using the $e m \times 3$ probe, and confirmed its expression in Dm at the adult stage (Fig. 5b, c). SAGFF70A;UAS:GFP and SAGFF120A;UAS:GFP fish expressed GFP in Dm at the adult stage, and in the dorsal telencephalon and part of the diencephalon at embryonic stages, recapitulating the expression pattern of the emx3 gene (Additional file 4: Figure S3). These results indicate that, in the SAGFF70A and SAGFF120A lines, Gal4FF is expressed under the control of the emx3 enhancer(s). GFP expression in Dm was slightly stronger in SAGFF120A;UAS:GFP fish, in which the transposon construct was located closer to the $e m x 3$ gene than in SAGFF70A;UAS:GFP fish. Consistent with this, the deficit observed in active avoidance conditioning was more severe in SAGFF120A than that observed in SAGFF70A (Fig. 3b, c).

To further confirm the behavioral phenotype observed in SAGFF120A;UAS:zBoTxBLC:GFP transgenic fish, we performed blind experiments for active avoidance fear conditioning using wild type, SAGFF120A;UAS:GFP, and SAGFF120A;UAS:zBoTxBLC:GFP fish (Fig. 6), in which the fish identities were not known to the experimenter. In these experiments, sibling fish were used for SAGFF120A;UAS:GFP and SAGFF120A;UAS:zBoTxBLC:GFP fish. While wild type and SAGFF120A;UAS:GFP fish could perform the conditioned active avoidance efficiently (Fig. 6a, b), SAGFF120A;UAS:zBoTxBLC:GFP fish exhibited significantly reduced performance in the avoidance response (Fig. 6c, d). Additionally, this result excluded the possibility that the transposon insertion in the SAGFF120A transgenic fish itself, but not the 

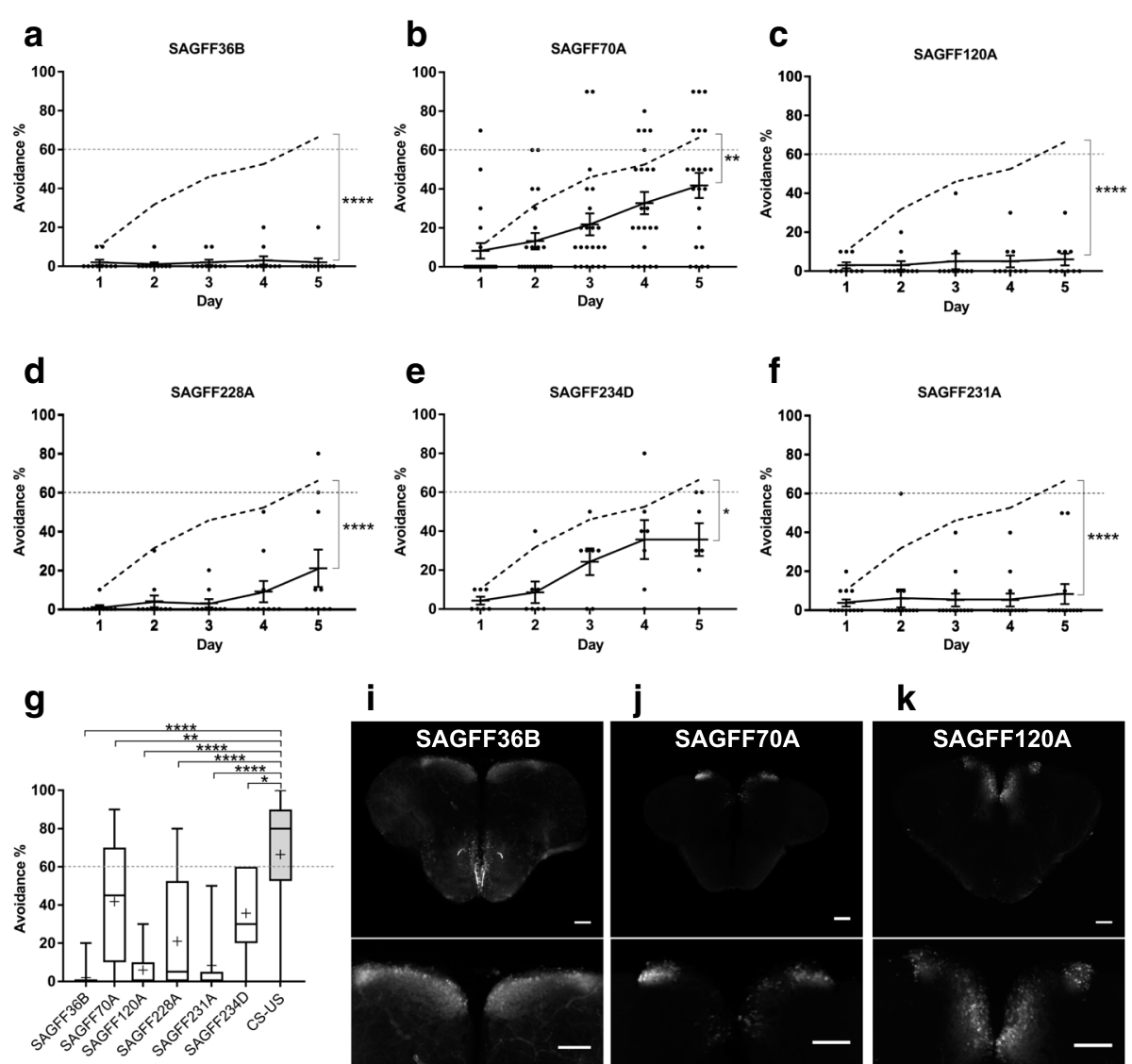

$\mathbf{k}$

h
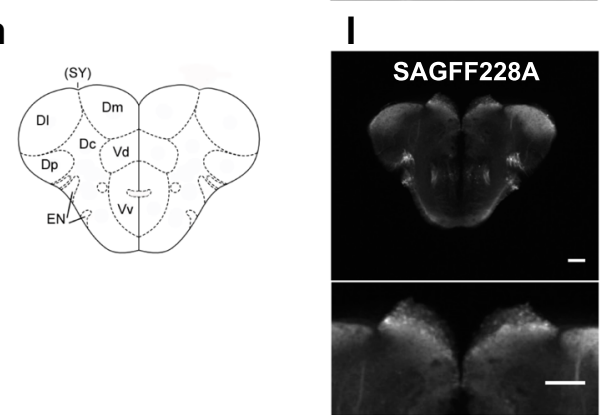

m
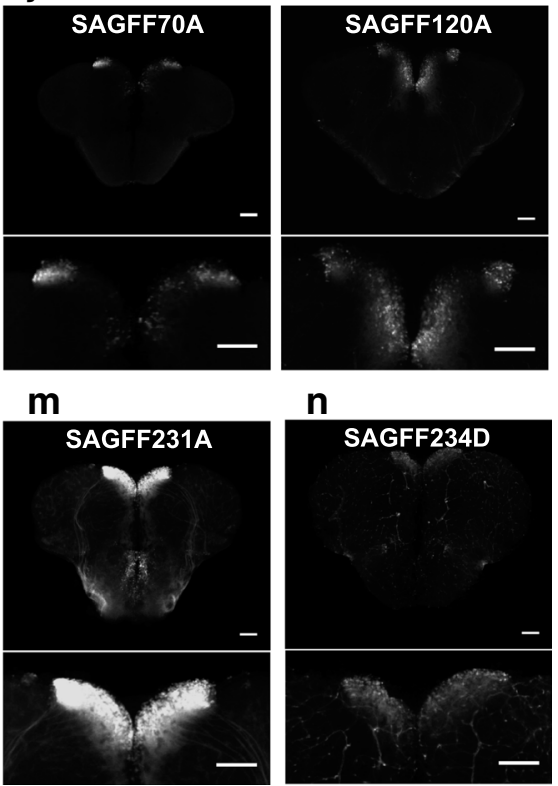

n

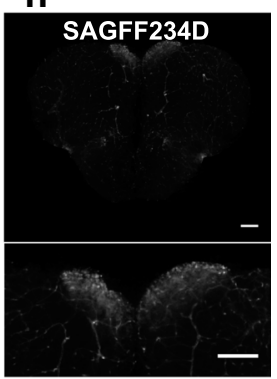

Fig. 3 Gal4FF transgenic fish lines that showed deficits in the active avoidance response and had expression patterns in the Dm. a-f Performance of two-way active avoidance response of wild type fish $(n=28)$ (Fig. 2 and shown in dotted lines) and double transgenic fish that are created by crossing the Gal4FF transgenic fish with the UAS:ZBoTxBLC:GFP fish. a SAGFF36B $(n=10)$. (b) SAGFF70A $(n=22)$. (c) SAGFF120A $(n=10)$. d SAGFF228A $(n=10)$. e SAGFF231A ( $n=13)$. f SAGFF234D $(n=11)$. Means \pm SEM and avoidance (\%) for individual fish are plotted. g Comparison of performance of avoidance responses at day 5 with Tukey box plot. Mean is marked by ' + '. Two-way ANOVA, fish groups (CS-US wild type, CS only wild type described in Fig. 2c, d and all double transgenic fish including fish described in Additional file 2: Figure S1) $\mathrm{x}$ training days (day 1, day 5), was performed $(F=7.236, P<0.0001$ ). Dunnett's multiple comparison post-hoc tests were performed between wild type and double transgenic fish on day $5 .{ }^{*} P<0.05,{ }^{* *} P<0.01$, ${ }^{* *} P<0.001$, and ${ }^{*}{ }^{*} * P<0.0001$. ns, not significant $(P>0.05)$. h A coronal view of the zebrafish telencephalon. Dm, medial zone of dorsal telencephalic area (D); Dl, lateral zone of D; Dc, central zone of D; Dp, posterior zone of D; SY, sulcus ypsiloniformis; $V d$, dorsal nucleus of ventral telencephalic area $(V ; V V$, ventral nucleus of $V$; EN, entopeduncular nucleus. i-n GFP expression patterns in the coronal section of the Gal4FF;UAS:GFP transgenic fish with magnified views of Dm. The Gal4FF transgenic fish are crossed with UAS:GFP effector fish. i SAGFF36B. j SAGFF70A. k SAGFF120A. I SAGFF228A. m SAGFF231A. n SAGFF234D. Scale bars, 200 um

neurotoxin gene expression induced by Gal4FF, was the cause for the behavioral deficits.

To examine whether SAGFF120A;UAS:zBoTxBLC:GFP fish had a deficit in acquisition of active avoidance conditioning, but not in possible consolidation processes during the night, we developed another experimental procedure, in which five sessions (20 trials for active avoidance conditioning per session) were conducted within 1 day. Additionally, with this procedure, wild type fish could perform the avoidance response in more than 


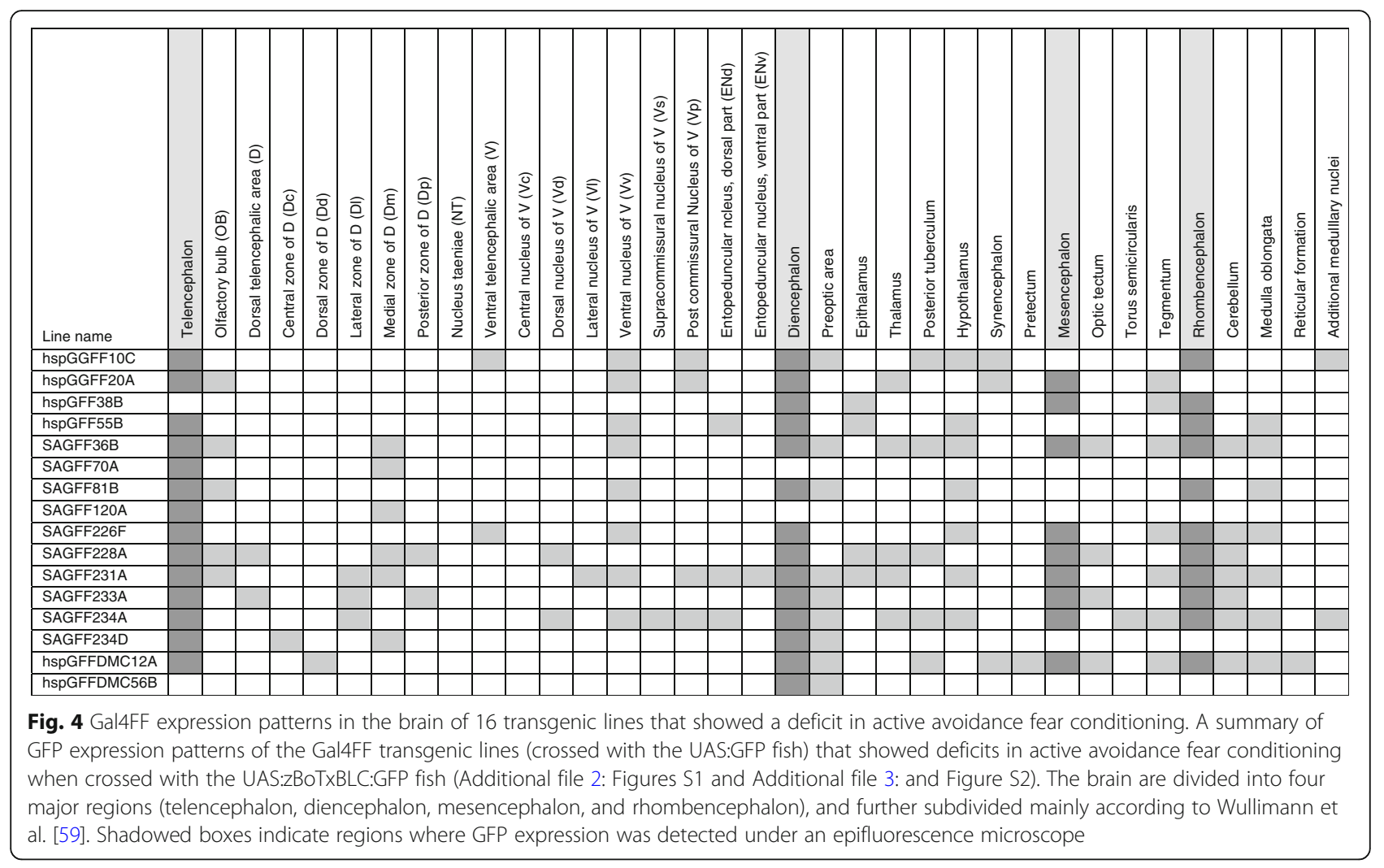

$60 \%$ of the trials after the second session, while SAGFF120A;UAS:zBoTxBLC:GFP fish exhibited a much reduced performance (Fig. 6e-g), indicating that double transgenic fish had a deficit prominently in the acquisition process.

\section{Other behavioral phenotypes in \\ SAGFF120A;UAS:zBoTxBLC:GFP fish}

To exclude the possibility that SAGFF120A;UAS:zBoTxBLC:GFP fish might have a deficit in the visual system, we analyzed the response to a light stimulus. Wild type fish showed increased swimming speed immediately after the CS (green LED) was turned on (within $100 \mathrm{~ms}$ ) (Fig. 7a). A similar light response was observed in SAGFF120A;UAS:zBoTxBLC:GFP fish (Fig. 7b, c), indicating that the double transgenic fish could detect the green LED.

To exclude the possibility that SAGFF120A;UAS:zBoTxBLC:GFP fish might have a deficit in the motor system, we analyzed the locomotor activity during free swimming of wild type and double transgenic fish. We detected comparable levels of locomotor activity in both wild type and double transgenic fish (Fig. 7d).

The mammalian amygdala mediates both Pavlovian and active avoidance fear conditioning $[1,2]$. Pavlovian

Table 1 Transposon integration sites in transgenic lines with Gal4FF expression in Dm

\begin{tabular}{llll}
\hline Line name & Chromosome & Features & Trapped gene/ nearest gene \\
\hline SAGFF36B & Chr8 & intergenic & fgf17/ zgc:123194-001 \\
SAGFF70A & Chr14 & intergenic & emx3 \\
SAGFF120A & Chr14 & intergenic & emx3 \\
SAGFF228A & ND & ND & repetitive sequence \\
SAGFF231A & Chr6 & intergenic & si:dkey-166d12.2 \\
SAGFF234D & Chr5 & intron & orai2 \\
\hline
\end{tabular}

The transposon integration sites in transgenic lines that had Gal4FF expression in Dm were cloned by inverse PCR, sequenced and mapped on the zebrafish genome. fgf17: fibroblast growth factor 17. emx3: empty spiracles homeobox 3. si:dkey-166d12.2: RAP1 GTPase Activating Protein. orai2: ORAl calcium release-activated calcium modulator 2

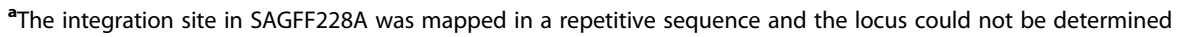



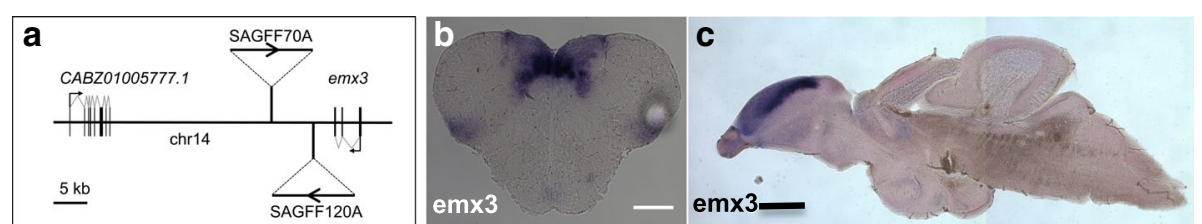

Fig. 5 SAGFF70A and SAGFF120A are enhancer trap lines of the emx3 gene. a Transposon integration sites in the SAGFF70A and SAGFF120A transgenic fish. $\mathbf{b}, \mathbf{c}$ In situ hybridization analysis of the adult brain using the emx3 probe. b Coronal section, scale bar $200 \mu \mathrm{m}$. c Sagittal section, scale bar $500 \mu \mathrm{m}$

fear conditioning has also been described in fish [10,11]. We tested whether SAGFF120A;UAS:zBoTxBLC:GFP fish also show a deficit in Pavlovian fear conditioning. In this procedure, we placed fish in a small rectangular tank and gave US (electric shock) $9 \mathrm{~s}$ after CS (light) was on. After repeating US coupling with CS five times, CS was administered and the turn activity was measured. We found that, while wild type fish showed an approximately three-fold increase in the turn activity after training, SAGFF120A fish exhibited significantly reduced turn activities (Fig. 7e and Additional file 5: Movie S2), indicating that the Gal4FF-expressing cells in SAGFF120A were essential for both active avoidance and Pavlovian fear conditioning.

In mammals, it has been reported that lesions in the basolateral amygdala lead to a deficit in an innate unconditioned response (freezing) to a natural dangerous stimulus (e.g., a ball of cat hairs for rats) [28]. It has been known that zebrafish display robust innate fear response to the alarm substance included in the skin extract, with fish exhibiting erratic movement followed by freezing $[29,30]$. To test whether

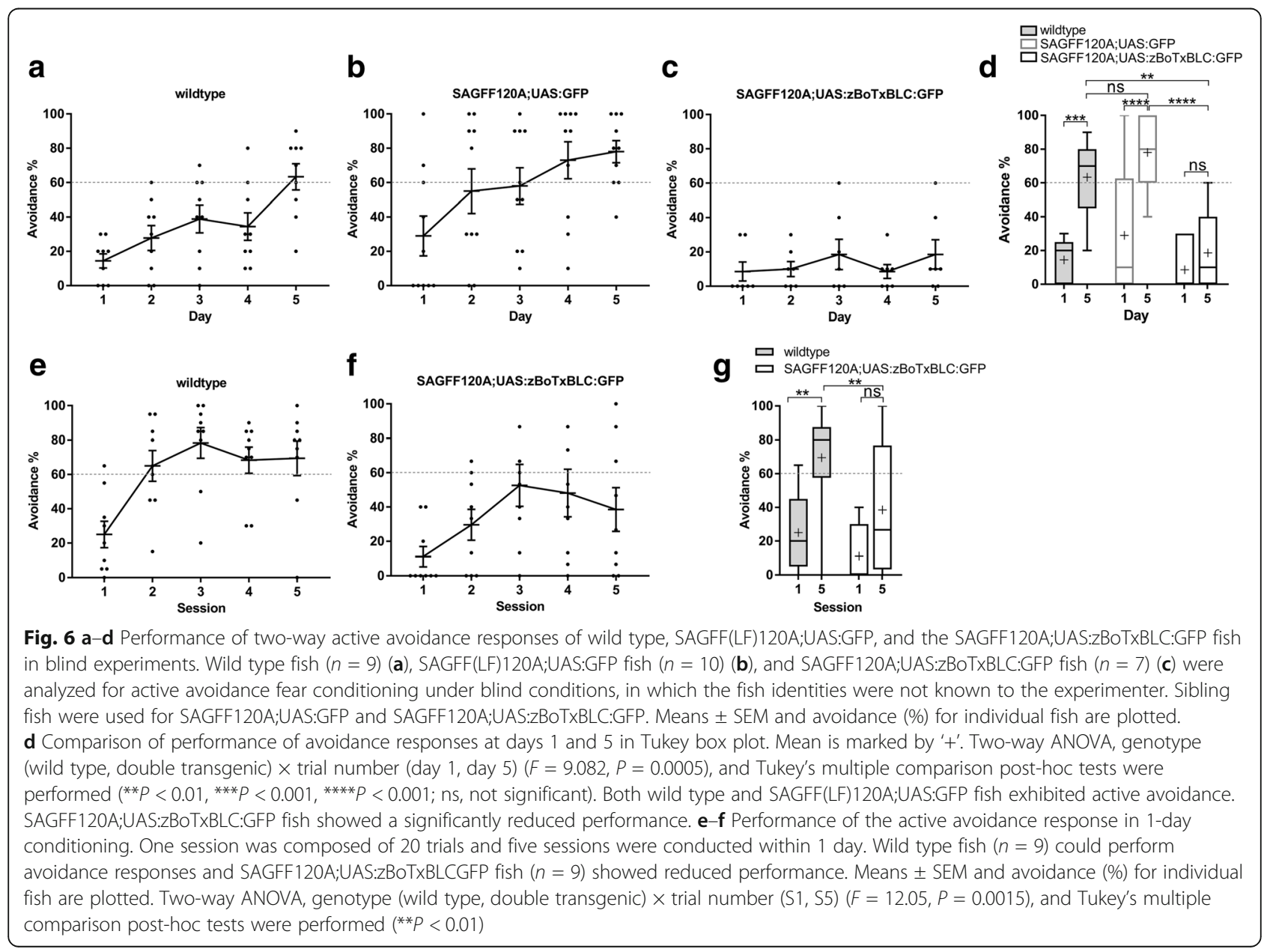




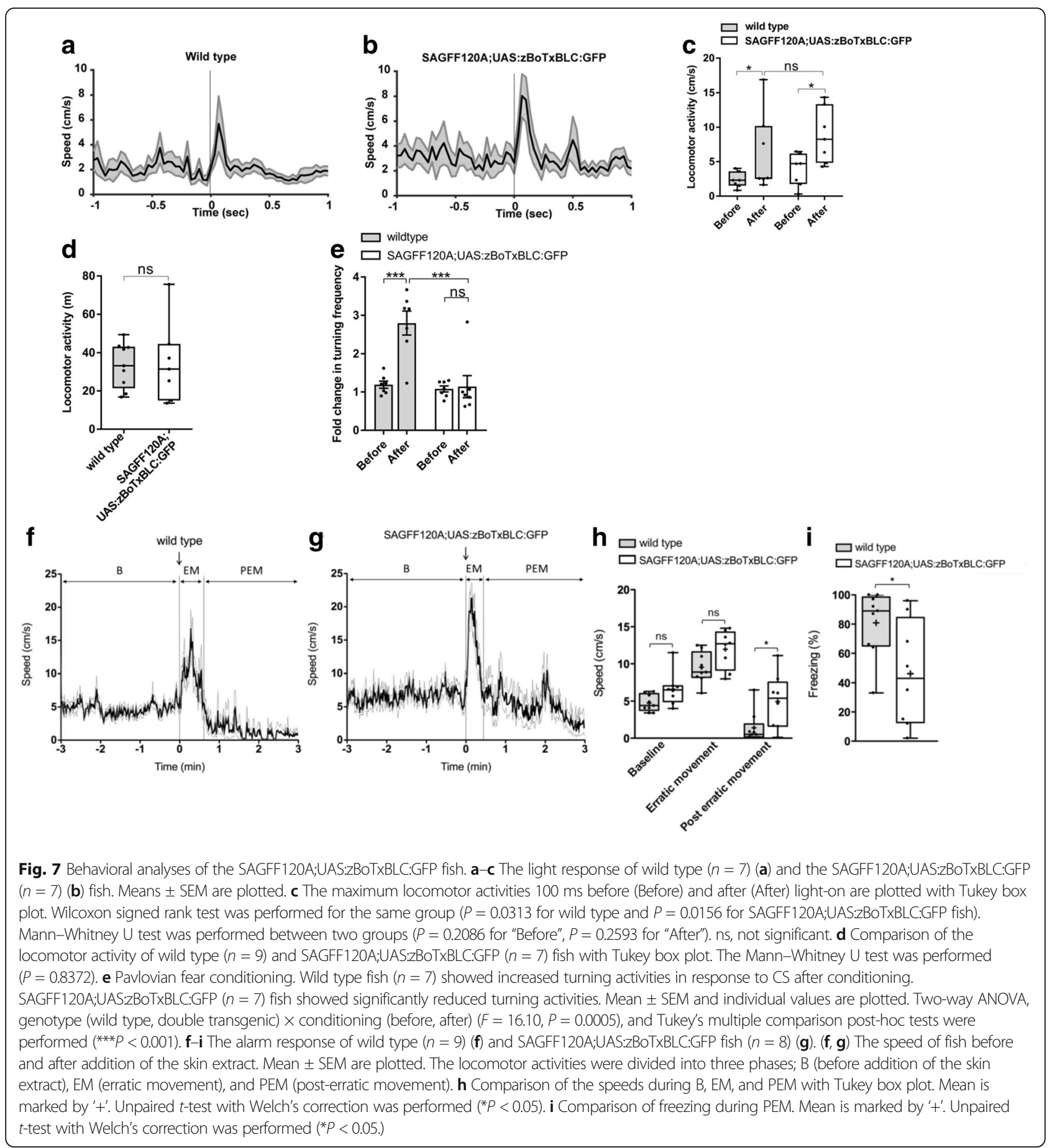

SAGFF120A;UAS:zBoTxBLC:GFP fish exhibit the alarm response, we analyzed locomotor activities of wild type $(n=9)$ and SAGFF120A;UAS:zBoTxBLC:GFP $(n=8)$ fish upon administration of the skin extract (Fig. $7 \mathrm{f}-\mathrm{i}$ ). Both wild type and double transgenic fish responded to the skin extract, and exhibited erratic movement (1.8- to 2.0-fold increase of the average speed) followed by freezing (Additional file 6: Movie
S3). However, we detected significant differences in the average speed and freezing duration in the phase of post-erratic movement between wild type and SAGFF120A;UAS:zBoTxBLC:GFP fish; namely, SAGFF120A;UAS:zBoTxBLC:GFP fish showed an increased average speed and decreased freezing duration, suggesting that Gal4FF-expressing cells may play a role in modulation of the freezing behavior (Fig. 7h, i). 

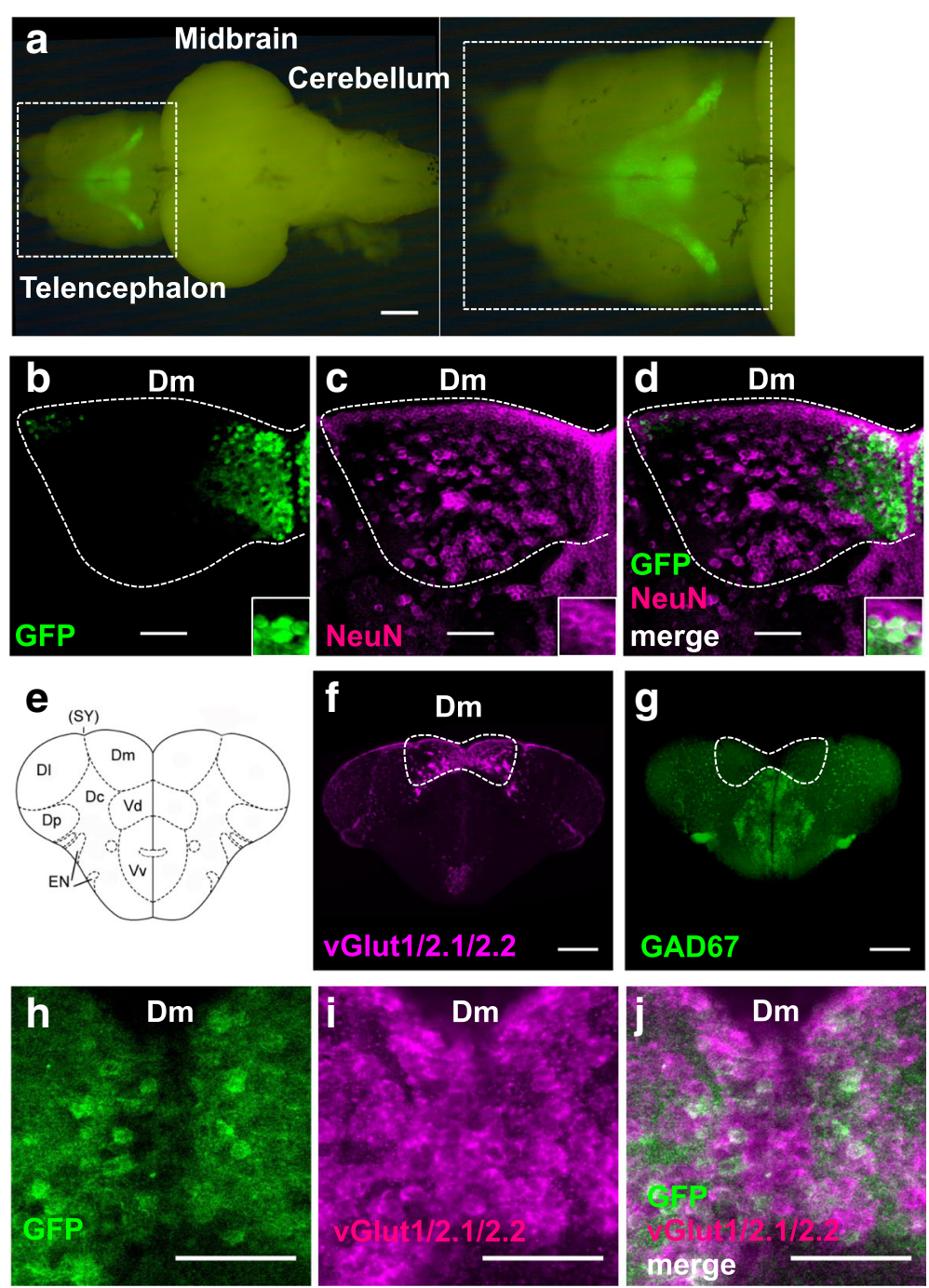

Fig. 8 Immunohistochemical analyses of the 120A-Dm neurons.

a Fluorescence imaging of the brain of SAGFF120A;UAS:GFP fish from the dorsal side. Dm, medial zone of the dorsal telencephalic area. Scale bars, $500 \mu \mathrm{m}$. b-d Double immunofluorescence staining of the telencephalon of SAGFF120A;UAS:GFP fish using the anti-GFP (green) (b) and anti-NeuN (magenta; neuronal marker) (c) antibodies. d A merged image; 16\% (1986/12282) of NeuN-positive cells were GFP-positive and 99\% (2394/2423) of GFP-positive cells were NeuN-positive. e A coronal view of the zebrafish telencephalon. Dm, medial zone of dorsal telencephalic area (D); DI, lateral zone of D; Dc, central zone of D; Dp, posterior zone of D; SY, sulcus ypsiloniformis; Vd, dorsal nucleus of ventral telencephalic area $(V) ; V$, ventral nucleus of $V ; E N$, entopeduncular nucleus. $\mathbf{f}$ Fluorescence in situ hybridization using the vglut1/2.1/2.2 probes. $\mathbf{g}$ Fluorescence in situ hybridization using the GAD67 probe. $\mathbf{h}-\mathbf{j}$ Immunofluorescence staining of the telencephalon of SAGFF120A;UAS:GFP fish using anti-GFP (green) (h) and fluorescence in situ hybridization using vglut1/2.1/2.2 probes (magenta) (i). j A merged image; 94\% (352/374) of GFP-positive cells were glutamatergic. Scale bars, $200 \mu \mathrm{m}(\mathbf{f}, \mathbf{g})$ and $50 \mu \mathrm{m}(\mathbf{b}-\mathbf{d}, \mathbf{h}-\mathbf{j})$

\section{Characterization of the neuronal population in Dm (120A-Dm neurons) essential for fear conditioning} The GFP expression pattern in the SAGFF120A;UAS:GFP line showed characteristic features from a dorsal view; one group of GFP-positive cells was located in the ventromedial region, and the other was located in the dorsal region and extended laterally towards the posterior telencephalon (Fig. 8a).

To examine if GFP-positive cells are indeed neurons, we analyzed brain sections from SAGFF120A;UAS:GFP fish by immunohistochemistry using anti-GFP and antiNeuN (neuronal marker) antibodies. Overall, 99\% of GFP-positive cells were NeuN-positive (2394/2423), revealing that most of the GFP-positive cells were neurons (Fig. 8bd-d). We also found that 16\% (1986/12282) of the NeuN-positive cells in the Dm area were GFP-positive, indicating that only a subpopulation of neurons in Dm were labeled in the SAGFF120A line. The GFP-positive (Gal4FF-positive) cells in the Dm area in the SAGFF120A line are hereafter referred to as 120A-Dm neurons. 


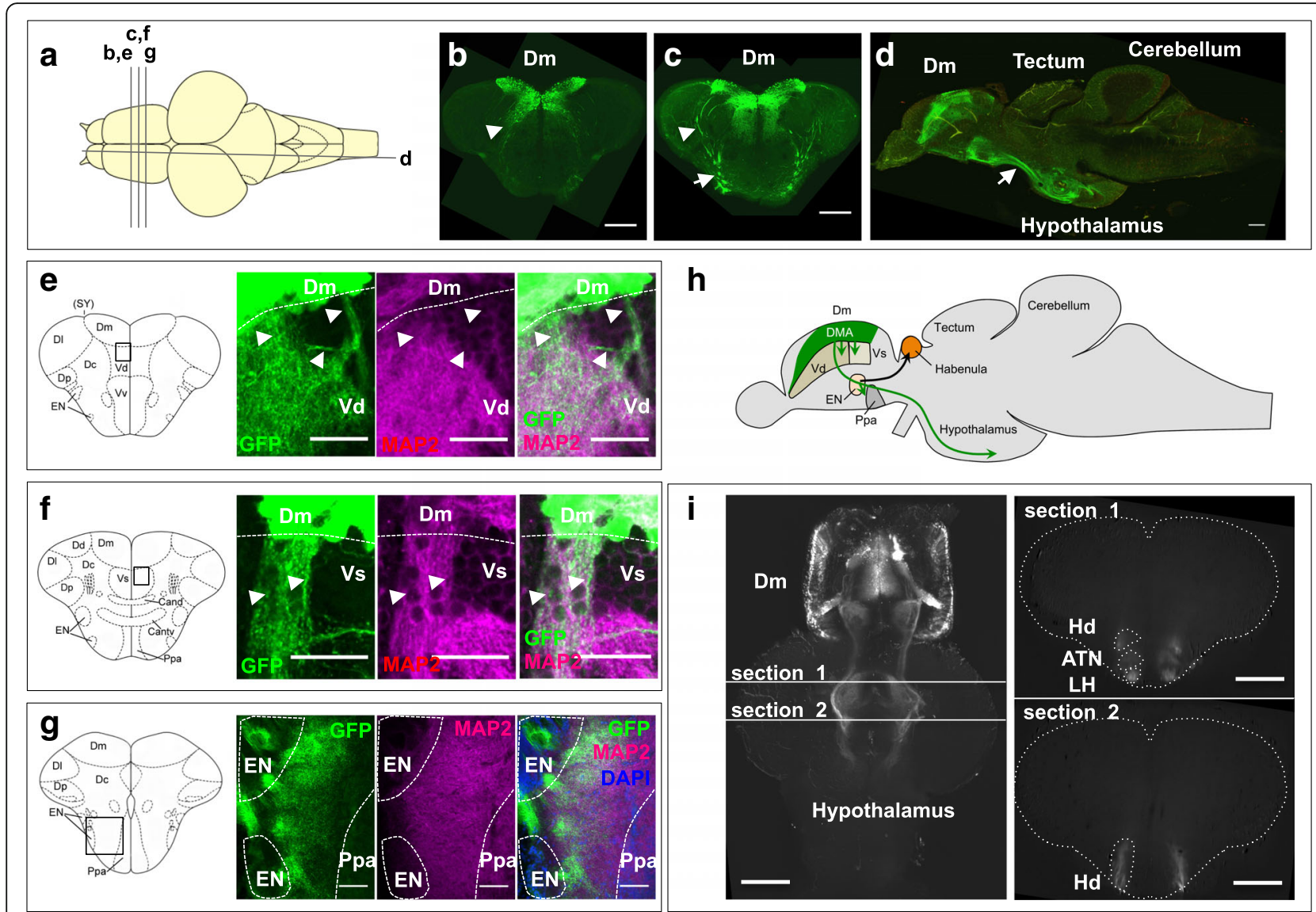

Fig. 9 Projections of 120A-Dm neurons. a Dorsal view of the brain. The positions of the sections are shown as bars (b-g). $\mathbf{b}-\mathbf{d}$ Immunofluorescence staining using anti-GFP. b, c Coronal sections of SAGFF70A;UAS:GFP;UAS:ZBOTxBLC:GFP fish. $\mathbf{d}$ Sagittal section of SAGFF120A;UAS:GFP;UAS:ZBOTxBLC:GFP fish. Axons of the 120A-Dm neurons project to the hypothalamus area. Arrowheads in $\mathbf{b}$ and $\mathbf{c}$ indicate projections from two groups of the 120A-Dm neurons. Arrows in $\mathbf{c}$ and $\mathbf{d}$ indicate the lateral forebrain bundle. $\mathbf{e}-\mathbf{g}$ Double immunofluorescence staining using anti-GFP (green) and anti-MAP2 (magenta; dendritic marker) of the brain of SAGFF120A;UAS:GFP fish. Schemes of coronal views of the telencephalon are shown on the left. e Projections to Vd. f Projections to Vs. e, $\mathbf{f}$ Arrowheads mark projections that were not co-stained with anti-MAP2. $\mathbf{g}$ Projections to the neuropil area of EN and Ppa. Cell bodies in EN and Ppa were stained with DAPI (blue). Dm, medial zone of dorsal telencephalic area (D); DI, lateral zone of D; Dc, central zone of D; Dp, posterior zone of D; SY, sulcus ypsiloniformis; Vd, dorsal nucleus of ventral telencephalic area (V); VV, ventral nucleus of V; EN, entopeduncular nucleus; Ppa, Parvocellular preoptic area; Cand, Commissura anterior, pars dorsalis; Cantv, Commissura anterior, pars ventralis. h A schematic view of axonal projections of the 120A-Dm neurons (green) to Vd, Vs, EN, Ppa, and the hypothalamus. i Light-sheet microscopy of a cleared brain from the SAGFF120A;UAS:GFP fish. Horizontal section (left) and coronal sections (right) showed projections of the 120A-Dm neurons terminated in the lateral hypothalamic nucleus (LH), the anterior tuberal nucleus (ATN), and dorsal zone of periventricular hypothalamus (Hd). Scale bars, $200 \mu \mathrm{m}(\mathbf{b}-\mathbf{d}), 50 \mu \mathrm{m}(\mathbf{e}, \mathbf{g}), 25 \mu \mathrm{m}(\mathbf{f})$, and $500 \mu \mathrm{m}$ (i)

We then performed in situ hybridization using the vglut1/2.1/2.2 and gad67 probes. In the zebrafish telencephalon, the pallium (dorsal telencephalon) and subpallium (ventral telencephalon) were rich in glutamatergic and GABAergic neurons, respectively (Fig. 8e-g), in agreement with previous reports $[14,15]$. We analyzed brain sections from SAGFF120A;UAS:GFP fish using the anti-GFP antibody and vglut1/2.1/2.2 probes, and found 94\% (352/374) of the GFP-positive cells to be glutamatergic (Fig. $8 \mathrm{~h}-\mathrm{j}$ ).

\section{Characterization of projections of the 120A-Dm neurons} To investigate projections of the 120A-Dm neurons, we first created serial cross sections and sagittal sections of the brain from SAGFF120A;UAS:GFP fish, and analyzed them by immunohistochemistry using the anti-GFP antibody and confocal microscopy (Fig. 9a-d). We found that the major projections from the 120A-Dm neurons proceeded ventrally within the telencephalon, joined the lateral forebrain bundle, proceeded more posteriorly, and terminated in the hypothalamic area (Fig. 9d). We also observed projections of the 120A-Dm neurons toward the subpallium area, including the dorsal nucleus (Vd) and the supracommissural nucleus of the ventral telencephalic area (Vs). Some of these projections were not co-immunostained with the anti-MAP2 antibody (dendritic marker) (Fig. 9e, f) [31], suggesting that they 


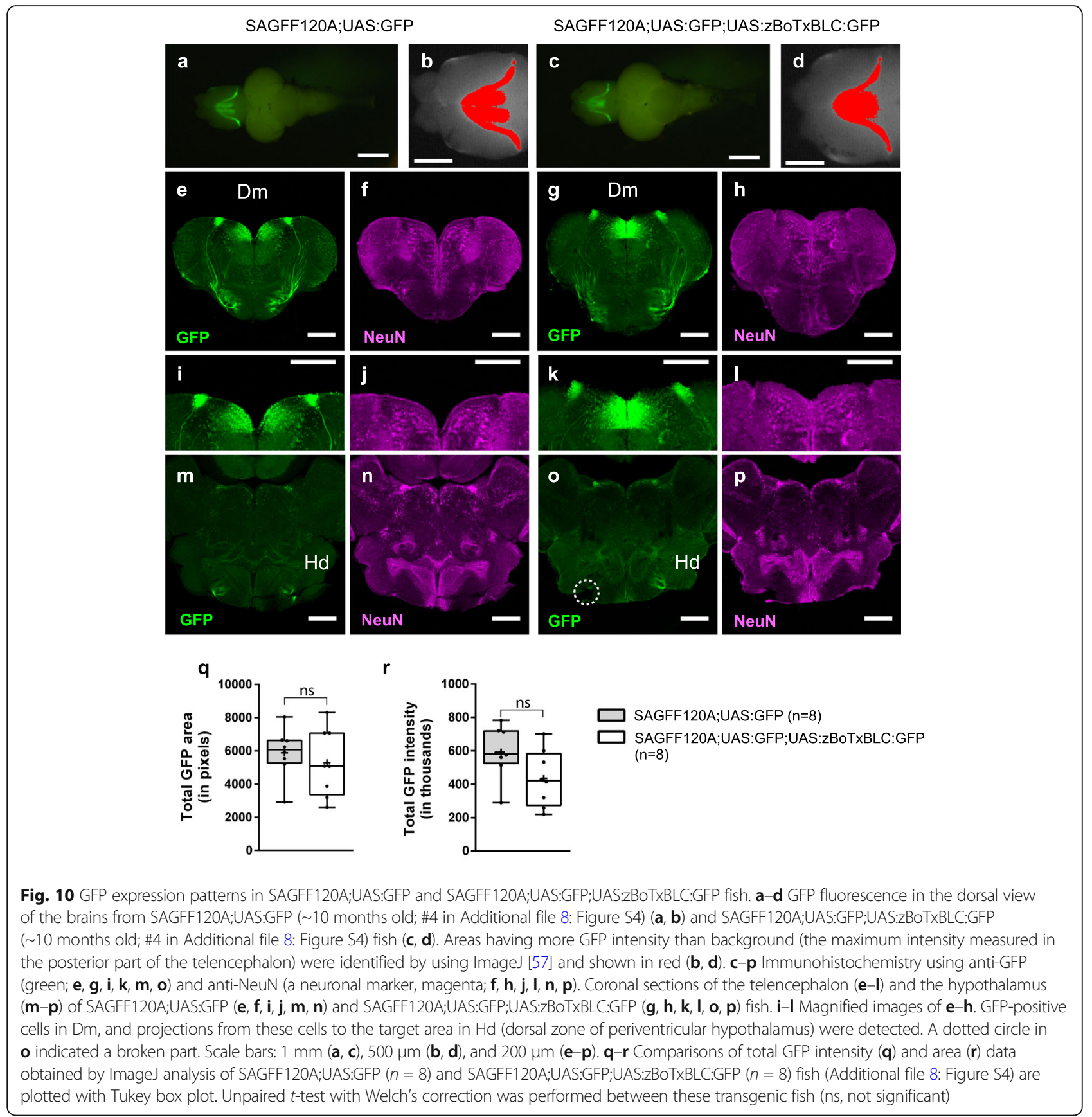

were axonal projections. In addition, we observed neurites of the 120A-Dm neurons spread in the neuropil areas of the entopeduncular nucleus (EN) and the preoptic area, suggesting connections to these nuclei (Fig. 9g, h).

Further, we prepared a cleared brain sample from SAGFF120A;UAS:GFP fish by the Scale method [32], and analyzed it with light-sheet microscopy. This analysis also visualized the projections from the Dm area to the ventral telencephalon and hypothalamus. In the hypothalamic area, the projections proceeded laterally and terminated in the lateral hypothalamic nucleus $(\mathrm{LH})$, the anterior tuberal nucleus (ATN), and dorsal zone of periventricular hypothalamus (Hd) (Fig. 9i and Additional file 7: Movie S4).

It should be noted that, in SAGFF120A;UAS:zBoTxBLC:GFP fish, the neurotoxin gene could be expressed from the larval to adult stages. To examine if any gross morphological differences were generated due to the possible continuous neurotoxin expression, we analyzed the brains from SAGFF120A;UAS:GFP and SAGFF120A;UAS:GFP;UAS:zBoTxBLC:GFP fish (Fig. 10, 
Additional file 8: Figure S4). First, we observed similar shapes of GFP expression patterns in the dorsal view of the telencephalon (Fig. 10a, c), and analyzed their intensities and the areas (Fig. 10b, d) and could not detect significant differences in the total GFP intensity and areas between these transgenic lines $(n=8$ for each transgenic lines; Fig. 10q, r). We then analyzed coronal sections from these brain samples by immunohistochemistry using GFP and NeuN antibodies, and could detect GFP-positive cells in the Dm area (Fig. 10e-l) and GFP-positive projections toward the ventral telencephalon from these cells that reached the target area in the $\mathrm{Hd}$ (dorsal zone of periventricular hypothalamus) in both of these transgenic lines (Fig. 10m-p). Further, the NeuN staining of these areas also showed similar patterns (Fig. 10f-p). Thus, we could not detect gross morphological differences in the GFP-positive and surrounding areas in SAGFF120A;UAS:GFP and SAGFF120A;UAS:GF$P ; U A S: z B o T x B L C: G F P$ fish at these levels of analysis.

\section{Discussion}

Functional and neurochemical similarities between the 120A-Dm neurons and the pallial amygdala nuclei

It has been postulated that the medial zone of the dorsal telencephalon $(\mathrm{Dm})$ in fish is a homolog of the mammalian amygdala based on neuroanatomical studies $[12,16,17]$ and ablation experiments in goldfish [18]. However, the neuronal population and circuitry had not yet been identified. In the present study, we performed a genetic approach using zebrafish and, for the first time, identified a subpopulation of neurons located in the Dm area, which we named 120A-Dm neurons, essential for acquisition of both active avoidance and Pavlovian fear conditioning.

The mammalian amygdala consists of pallial (cortical) and subpallial (striatal) portions, and is further subdivided into multiple nuclei. The BLA, which are included in the pallial portion, contain predominantly glutamatergic neurons and are essential for the CSUS association [3]; namely, it was shown that the BLA lesions caused deficits in both Pavlovian and active avoidance fear conditioning $[1,5,33]$. The 120A$\mathrm{Dm}$ neurons identified in the teleost $\mathrm{Dm}$ were also mostly glutamatergic and essential for both Pavlovian and active avoidance fear conditioning. From these functional and neurochemical similarities, we suggest that the 120A-Dm neurons are the functional equivalent of the pallial amygdala, and presumably neurons in BLA. It is not known whether the entire population of the 120A-Dm neurons or only part of them are essential for fear conditioning. Further subdivision of the 120A-Dm neurons will be required to answer this question.
In mammals, BLA lesions cause a deficit in an innate unconditioned response to a natural dangerous stimulus (for instance, cat hairs for rats) [28]. Additionally, there was a contradictory report describing that inactivation of BLA impaired the learned, but not innate, fear response in rats [34]. Herein, we tested whether the 120A-Dm neurons are involved in the innate fear response in zebrafish by analyzing reactions to a skin extract $[29,30]$. Similarly to wild type fish, SAGFF120A;UAS:zBoTxBLC:GFP fish could respond to the skin extract and perform erratic movement and freezing. However, during the post-erratic movement phase, fish showed reduced freezing behaviors in comparison to wild type fish, suggesting that $120 \mathrm{~A}-\mathrm{Dm}$ neurons may play a role in modulation of the freezing behavior. Consistent with this, it was reported that cells in the Dm are activated upon administration of a skin extract [35]. Further, it has been shown that the alarm response- or alarm substance-induced fear conditioning can be modulated by social buffering [36] or administration of the endocannabinoid receptor CB1 agonist [35]. Transgenic fish that expressed Gal4FF in 120A-Dm neurons should allow us to investigate a neuronal basis of these behaviors as well as other motivational and emotional behaviors in zebrafish, such as light/dark choice or drugseeking behaviors $[19,20]$, that are thought to be mediated by the amygdala-like functions of Dm.

In addition to the Dm, the present study highlighted other forebrain regions possibly important for fear conditioning. For instance, we found a relatively large number of lines that showed reduced avoidance responses and that commonly had Gal4FF expression in the ventral nucleus of the ventral telencephalon $(\mathrm{Vv})$ or the preoptic area. $\mathrm{Vv}$ has been postulated to be a homolog of the septal nuclei of mammals $[13,37]$, which also play a crucial role in fear learning [38]. The preoptic area has been postulated to be a homolog of the mammalian paraventricular nucleus of the hypothalamus (PVN) [39], containing the magnocellular neurosecretory system that mediates fear responses [40]. hspGFFDMC56B fish had rather specific Gal4FF expression in the preoptic area and should be used for further studies to explore their role in fear conditioning.

emx3-expressing neurons are essential for fear conditioning In the SAGFF70A and SAGFF120A lines, Gal4FF was expressed in a pattern similar to that of the $e m \times 3$ gene. The zebrafish $e m x 3$ gene is expressed in the dorsal telencephalon at the embryonic stage and in the dorsomedial region in the adult brain [25-27]. Knockdown of the $e m \times 3$ function by morpholino impairs expression of dorsal telencephalic marker genes [41]. However, the function of the emx3-expressing cells had not been analyzed. The present study revealed the role of the emx3- 
expressing neurons in fear conditioning. It should be noted that, in our approach, the botulinum neurotoxin was continuously expressed throughout development. Although we could not detect gross morphological changes in the GFP-positive neurons in SAGFF120A;UAS:GFP;UAS:zBoTxBLC:GFP fish, a conditional system that prohibits neuronal activities only in the adulthood is required to examine the possibility that the toxin expression during developmental stages may have caused the observed behavioral deficits. Efforts are currently in progress along this line.

The mouse genome has two paralogs, $E m \times 1$ and $E m \times 2$ [42]. Emx1 is expressed in the dorsal telencephalon in the developing brain, and Emx1-expressing cells give rise to excitatory neurons in the pallium, including pallial portions of the amygdala [43]. Emx2 is expressed earlier and more broadly, and plays a major role in the formation of the medial limbic cortex [44]. The Emx1-expressing cells in the developing telencephalon in chicken and Xenopus also contribute to cells in amygdalar nuclei $[45,46]$. Thus, roles of cells expressing the emx family genes in the developing brain may be conserved during vertebrate evolution. However, the function of Emx-expressing cells in the adult brain was not characterized. It should be interesting to investigate $E m x$-expressing neurons in the adult brain in other vertebrates to see whether those neuronal populations harbor essential roles in fear conditioning as well.

\section{Projections of 120A-Dm neurons to the hypothalamus}

We found that 120A-Dm neurons had major projections to the hypothalamic area. In mammals, the hypothalamus is important in fear responses, controlling heart rate and blood pressure $[1,2]$. We assume that the Dmhypothalamus connection should also play an important role in mediating fear responses in fish. In previous work using goldfish, efferent projections from Dm to the hypothalamic area, including the ATN and dorsal zone of periventricular $\mathrm{Hd}$, were identified by anterograde labeling [17], and minor outputs from Dm to the LH were detected by retrograde labeling [47]. The present study clearly visualized projections of 120A-Dm neurons that terminated in the hypothalamic area, including ATN, $\mathrm{LH}$, and Hd, consistently with the results obtained in the tracer experiments in goldfish.

In mammals, the CeA is the major output center, has projections to the lateral hypothalamus, and predominantly contains GABAergic neurons $[1,2,5]$. In contrast, $120 \mathrm{~A}-\mathrm{Dm}$ neurons are mostly glutamatergic and thought to be a functional equivalent of the pallial amygdala. Further studies on the roles of excitatory projections from Dm to the hypothalamus should provide new insights into the conservation and diversification of limbico-hypothalamic connections during evolution.

\section{Projections of 120A-Dm neurons to the other telencephalic} regions

We found the projection of 120A-Dm neurons also terminated in the neuropil area of the EN and preoptic area, Vd, and Vs, suggesting that these are possible targets. The EN consists of a dorsal GABAergic part and a ventral glutamatergic part, which have been hypothesized to be homologous to the EN of non-primate mammals (internal segment of the globus pallidus of primates) and the bed nucleus of the stria medullaris, respectively [14]. In goldfish and zebrafish, the ventral glutamatergic neurons have been shown to project to the $\mathrm{Hb}$ nuclei $[11,48]$, and it was recently shown that the $\mathrm{Hb}$-median raphe circuit in zebrafish is essential for active avoidance conditioning [11]. Thus, we hypothesize that the projection of $120 \mathrm{~A}-\mathrm{Dm}$ neurons to the EN may play a role in mediating active avoidance responses. Consistent with this hypothesis, hspGFF38B and hspGFF55B fish, which strongly expressed Gal4FF in the $\mathrm{Hb}$ and EN, respectively, also exhibited deficits in active avoidance conditioning when crossed with the UAS:neurotoxin line (Additional file 2: Figure S1). Further analyses using these transgenic fish should reveal the role of the Dm-EN-Hb circuit in active avoidance responses. Vd and Vs are structures located in the subpallium and rich in GABAergic neurons, and have been hypothesized as homologs of the mammalian striatum and CeA, respectively [14]. Thus, these connections may correspond to connections of BLA to the striatum, and the intra-amygdaloid connection of BLA to CeA, which have been described in mammals [1, 2, 49].

\section{The genetic approach reveals functional neuronal circuits mediating adult behavioral phenotypes}

Herein, we succeeded in performing a genetic approach to study a learning behavior in zebrafish. We think this success mainly relies on three factors. Firstly, our trap lines expressed Gal4FF specifically and strongly in the adult brain. We have shown that Gal4FF is a strong but less-toxic transcription activator in zebrafish $[21,50]$ and, since then, performed gene and enhancer trap genetic screens to label specific cell types by Gal4FF expression at the larval stages [51]. The present study demonstrated that the gene and enhancer trap approaches are applicable to generate specific Gal4FF expression patterns at the adult stage as well. Secondly, the UAS:zBoTxBLC:GFP line expressed the neurotoxin reliably and reproducibly in combination with various Gal4FF lines. It was reported that some UAS effector lines, especially those containing 14xUAS, suffered from silencing effects [52]. In contrast, we have been using 5xUAS for UAS-effector lines [21], and have not experienced such severe silencing effects. Further, when we 
created UAS:zBoTxBLC:GFP fish, we generated more than 30 different insertions, selected transgenic fish that showed the strongest expression by crossing them with several different Gal4FF driver lines, and established the best line with a single transposon insertion. The UAS:zBoTXBLC:GFP line thus established worked effectively in the larval stages [24] as well as in the adult stage (this study). Thirdly, our protocol developed for active avoidance fear conditioning has worked very efficiently and reproducibly, enabling the identification of transgenic lines with reduced learning activities out of many candidate lines. In summary, the present study demonstrated that the genetic approach combined with a behavioral paradigm is powerful to dissect functional neuronal circuits in the adult zebrafish brain, and should be applicable to the study of other brain circuits and behaviors.

\section{Conclusions}

Fear conditioning is commonly observed in vertebrate species. In teleost, it has been postulated that the medial zone of the dorsal telencephalon $(\mathrm{Dm})$ is a homolog of the mammalian amygdala, and essential for retention of the conditioned avoidance responses. However, Dm is a broad area and functional neuronal populations had not yet been identified. Herein, we identified a subpopulation of neurons in Dm essential for fear conditioning through a genetic approach in zebrafish. These neurons are mostly glutamatergic and have projections to other brain regions, including the hypothalamic area and ventral telencephalon. We propose that these should be functional equivalents of neurons in the mammalian pallial amygdala, mediating a CS-US association. Thus, we established a basis for understanding the evolutionary conservation and diversification of functional neural circuits mediating fear conditioning in vertebrates.

\section{Methods}

\section{Fish}

Transgenic fish that expressed Gal4FF were generated by the gene trap and enhancer trap methods [21]. Transposon integration sites in transgenic fish lines were analyzed by Southern blot hybridization and inverse PCR as described previously [53]. UAS:GFP fish were used to visualize Gal4FF expression [21]. The T2SUASzBoTXBLCGFP construct containing 5xUAS, TATA sequence, the codon-optimized botulinum toxin $B$ light chain gene [54] fused to the EGFP gene, and SV40 polyA between cis-sequences of Tol2 was created and injected to fertilized eggs to generate the UAS:zBoTXBLC:GFP transgenic fish.

\section{Analysis of Gal4FF expression in the adult brain}

The GFP expression patterns in the adult brain were first observed under a fluorescence microscope ( $\mathrm{MZ}$ 16FA, Leica Microsystems). The heads were then fixed in $4 \%$ paraformaldehyde and dissected to take the brains out of the skulls as described previously [55]. The isolated brains were observed under a fluorescence microscope (MZ 16FA, Leica Microsystems). The fixed brain samples were embedded in $1 \%$ agarose in $0.1 \mathrm{M}$ phosphate buffered saline (PBS; pH 7.4) and $100 \mu \mathrm{m}$-thick serial coronal sections were made by using a vibratome. The slices were collected in 24-well plates and mounted on slide glasses (Matsunami) using PermaFluor Aqueous Mounting Medium (Thermoscientific). Sections were observed under an upright epifluorescence microscope (Axio Imager Z1, Zeiss).

\section{Preparation of fish for behavioral analyses}

Fish aged from 5 months to 1.5 years were used for behavioral studies. Prior to behavioral assays, fish were moved to a behavioral assay room and kept in isolation in 2-L tanks for 2 days.

\section{Two-way active avoidance fear conditioning (5-day procedure)}

A white opaque acrylic tank (length $41 \mathrm{~cm} \times$ width 17 $\mathrm{cm} \times$ height $12 \mathrm{~cm}$ ) with transparent walls at both ends, a trapezoidal wedge $(10 \mathrm{~cm}$ at the top and $20 \mathrm{~cm}$ at the bottom $\times$ width $17 \mathrm{~cm} \times$ height $5 \mathrm{~cm}$ ) in the center of the tank, green LEDs (3.3 V DC, $2 \mathrm{~A})$, and a pair of platinum mesh electrodes ( $12 \mathrm{~V} \mathrm{AC}$ ) were used. Behaviors were monitored and analyzed by programs created by using LabView 8.6 (National Instruments). Habituation was performed for $15 \mathrm{~min}$ per day for 2 days in the shuttle box. Conditioning involved (1) $2 \mathrm{~min}$ in the shuttle box; (2) initiating CS (green LED) and US $10 \mathrm{~s}$ later (12 V AC electric shock); and (3) turning off of CS and US 5 $\mathrm{s}$ after initial US; (4) if fish escaped while CS was on, US was not given; and (5) if the fish moved to another compartment while US was on, both CS and US were turned off. The process was repeated 10 times with an intertrial interval of $25 \pm 5$ s per day on 5 consecutive days. The avoidance index was calculated as the number of successful escape responses per 10 trials. The two-way active avoidance conditioning was also performed as blind experiments, in which the fish identities were not known to the experimenter (Fig. 6).

\section{Two-way active avoidance fear conditioning (1-day procedure)}

The same setup as the 5-day procedure was used. Habituation was performed for $1 \mathrm{~h}$ per day for 2 days in the shuttle box. Conditioning involved (1) $5 \mathrm{~min}$ in the shuttle box; (2) initiating CS and US $10 \mathrm{~s}$ later (9 V AC 
electric shock); (3) turning off of both CS and US $5 \mathrm{~s}$ after US; (4) if fish escaped while CS was on, US was not given; and (5) if the fish moved to another compartment while US was on, both CS and US were turned off. The process was repeated 20 times with intervals of $25 \pm 5$ s per session, and five sessions were performed with an inter-trial interval of $3 \mathrm{~min}$. The avoidance index was calculated as the number of successful escape responses per 20 trials.

\section{The light response}

The response to a light stimulus, which was described previously for larval zebrafish [56], was measured by using adult fish. A white opaque acrylic box (length 12 $\mathrm{cm} \times$ width $17 \mathrm{~cm} \times$ height $12 \mathrm{~cm}$ ) with a transparent wall on one-side and equipped with a green LED light $(3.3 \mathrm{~V}, 2 \mathrm{~A} \mathrm{DC})$ was used. The water level was $5 \mathrm{~cm}$ in depth. Fish behavior was monitored and analyzed by programs created using LabView 8.6 (National Instruments). Fish were kept in the apparatus for $10 \mathrm{~min}$ and the green LED light was then turned on for $10 \mathrm{~s}$. Fish locomotion was recorded at $27 \mathrm{fps}$, and the locomotor activities (speed) of the fish $100 \mathrm{~ms}$ before and after light-on were analyzed. Movie analysis was carried out with ImageJ 1.48v (US National Institute of Health) [57].

\section{Locomotor activity}

Fish were habituated for $5 \mathrm{~min}$ in an opaque tank (length $33 \mathrm{~cm} \times$ width $19 \mathrm{~cm} \times$ height $15 \mathrm{~cm}$ ). Behaviors were recorded for $10 \mathrm{~min}$ and analyzed by using a program created with LabView 8.6 (National Instruments). Locomotor activities were calculated as distances travelled in $10 \mathrm{~min}$.

\section{Pavlovian fear conditioning}

A white opaque acrylic box (length $12 \mathrm{~cm} \times$ width 17 $\mathrm{cm} \times$ height $12 \mathrm{~cm}$ ) with a transparent wall on one-side equipped with a green LED light (3.3 V, 2A DC) and a pair of platinum mesh electrodes (9 V AC) was used. Behaviors were monitored and analyzed by programs created with LabView 8.6 (National Instruments). Habituation was performed by (1) placing fish for $10 \mathrm{~min}$ in the tank, (2) then initiating CS for $10 \mathrm{~s}$, five times, at 50-s intervals, (3) followed by $10 \mathrm{~min}$ of free-swimming. Conditioning involved initiating CS and, $9 \mathrm{~s}$ later, initiating US for $1 \mathrm{~s}$, followed by turning off of both CS and US. The process was repeated 5 times at 50-s intervals and the entire conditioning process was repeated once. The test involved (1) 10 min of free-swimming after training and (2) delivery of CS five times at 50-s intervals. The behavior was recorded at $27 \mathrm{fps}$ and the movies were analyzed with ImageJ [57]. Turning angles were determined by measuring the change of the head-tail axis of the fish. Turning with angles greater than $90^{\circ}$ within six frames $(0.22 \mathrm{~s})$ was defined as a conditioned response. The number of conditioned responses during the $10 \mathrm{~s}$ before and after CS was counted, and fold changes in turning frequencies were calculated with or without conditioning.

\section{The alarm response}

Skin extracts were prepared fresh and kept on ice on the day of use [30]. Adult fish ( $>3$ months old) were anesthetized in Tricaine $(0.025 \%)$ and quickly sacrificed by decapitation. Excess water was removed from the skin using a paper towel, and 15 shallow cuts were made on each side of the trunk, avoiding contamination of the blood. Cuts were washed with distilled water, and $10 \mathrm{~mL}$ of skin extract were collected from each fish. To test alarm response, fish were habituated for $10 \mathrm{~min}$ in a $2-\mathrm{L}$ tank (length $25 \mathrm{~cm} \times$ width $6.5 \mathrm{~cm} \times$ height $16 \mathrm{~cm}$ ) equipped with delivery tubes at both ends. Then, $2 \mathrm{~mL}$ of the skin extract was applied to the tank. Behavior was recorded for $3 \mathrm{~min}$ before and after addition of the skin extract. The locomotor activity of wild type and the double transgenic fish was divided into three phases, namely baseline (B, before addition of the skin extract), erratic movement (from addition of the skin extract to the time when the average speed dropped to the baseline level; $38 \mathrm{~s}$ for wild type and $24 \mathrm{~s}$ for the double transgenic fish), post-erratic movement, and analyzed. Freezing was defined as locomotor activities with a speed of less than $5 \mathrm{~mm} / \mathrm{s}$. The movies were analyzed using Image [57].

\section{Statistical analyses}

For active avoidance performance (5-day procedure), analysis of variance (ANOVA) and Dunnet's multiple comparison tests were performed to test the statistical significance between test samples and control. For active avoidance performance (1-day procedure), ANOVA and Tukey's multiple comparison tests were performed to test the statistical significance between test samples and control. For locomotive activity, Kruskal-Wallis test was performed. In Pavlovian fear conditioning, two-way ANOVA and Tukey's multiple comparison post-hoc tests were performed. No data points were excluded in these analyses. All the statistical tests were performed by using PRISM 6 (GraphPad software).

\section{Immunohistochemistry and in situ hybridization}

Coronal/sagittal sections of $100 \mu \mathrm{m}$ in thickness were used for immunohistochemistry. The samples were treated for $1 \mathrm{~h}$ at room temperature in blocking buffer, $0.5 \%$ Tween-20 or Triton X-100, 3\% bovine serum albumin (Sigma) in PBS, and then incubated overnight with primary antibodies diluted in blocking buffer at $4{ }^{\circ} \mathrm{C}$. The samples were then washed with $0.5 \%$ Tween- 20 or Triton X-100 in PBS and incubated with secondary antibodies. Rabbit anti-GFP polyclonal (1:500 dilution, 
A6455; Invitrogen, RRID:AB_221570, LOT number = $1,650,113)$, mouse anti-NeuN monoclonal (1:200 dilution, MAB377; Millipore, RRID:AB_2298772, LOT number $=2,428,671)$, and mouse monoclonal anti-MAP2 antibody [AP-20] (1:300 dilution, ab11268; Abcam, RRID:AB_297886) were used for the primary antibodies. AlexaFluor 488 goat anti-rabbit IgG (1:400 dilution, A11008; Invitrogen, RRID:AB_143165) and AlexaFluor 633 goat anti-mouse IgG (1:1000 dilution, A21050; Invitrogen, RRID:AB_141431) were used for the secondary antibodies. For in situ hybridization, digoxigenin-labeled probes were synthesized by using the $e m \times 3$ and the vglut 1/2.1/2.2 cDNAs as templates. In situ hybridization was performed on coronal slices of fixed brain using a protocol described previously [58] with modifications. Prehybridization and hybridization were performed at 65 ${ }^{\circ} \mathrm{C}$ for $2 \mathrm{~h}$ in $\mathrm{Hyb}(+)$ solution or at $65{ }^{\circ} \mathrm{C}$ overnight in $\mathrm{Hyb}(+)$ solution containing approximately $100 \mathrm{ng}$ of digoxigenin-labeled probes, respectively. The samples were washed with $66 \% \mathrm{Hyb}(-) / 2 \mathrm{X}$ SSCT at $65{ }^{\circ} \mathrm{C}$ for 30 min, $33 \% \mathrm{Hyb}(-) / 2 \mathrm{X} \mathrm{SSCT}$ at $65{ }^{\circ} \mathrm{C}$ for $30 \mathrm{~min}, 2 \mathrm{X}$ SSCT at $65^{\circ} \mathrm{C}$ for $15 \mathrm{~min}$, and with $0.2 \mathrm{X}$ SSCT at $65^{\circ} \mathrm{C}$ for $30 \mathrm{~min}$ twice. $\mathrm{Hyb}(-)$ : $50 \%$ formamide, $5 \mathrm{X}$ SSC (Gibco), 0.1\% Tween-20 (Pierce). $\operatorname{Hyb}(+)$ : $\mathrm{Hyb}(-)$ with 5 $\mathrm{mg} / \mathrm{mL}$ RNA purified from torula yeast (Sigma) and 50 $\mu \mathrm{L} / \mathrm{mL}$ heparin (Sigma). The samples were then incubated in blocking solution (2\% blocking reagent in PBST) (Roche) at $4{ }^{\circ} \mathrm{C}$ overnight and then incubated in 1:5000 dilution of anti-digoxigenin-AP Fab-fragments (11093274910; Roche, RRID:AB_514497) at $4{ }^{\circ} \mathrm{C}$ overnight. For $e m x 3$, signals were detected using BM purple (Roche). The reaction was stopped by washing with PBST. The slices were mounted on slide glasses (Matsunami) using glycerol-gelatin mounting medium and observed under a microscope (Imager Z1). Images were taken with an AxioCam MRc5 (Zeiss) camera and analyzed with Axio Vision Ver4.1 imaging software (Zeiss). For vglut1/2.1/2.2, signals were detected with Fast Red Tablets (Roche). The slices were mounted on slide glasses using PermaFluor Aqueous Mounting Medium (Thermoscientific) and observed with a laser scanning confocal microscope (FV1000-D; Olympus) or a Zeiss confocal microscope with Yokogawa CSU-W1 laser scanning unit (Yokogawa). Images were processed with ImageJ [57].

\section{Preparation of a cleared brain and light-sheet microscopy} The Scale method [32] was applied in the preparation of a cleared brain from SAGFF120A;UAS:GFP fish. The head was fixed with $4 \%$ paraformaldehyde/PBS (Wako) at $4{ }^{\circ} \mathrm{C}$ for overnight, and then dissected. The brain sample was washed with PBS, incubated in $20 \%$ sucrose/PBS at $4{ }^{\circ} \mathrm{C}$ for 2 days, embedded in OCT compound (Sakura), and frozen with liquid nitrogen. The sample was then thawed and washed with PBS, transferred into the ScaleA2 solution, and kept at $4{ }^{\circ} \mathrm{C}$ for more than 3 weeks. The ScaleA2 solution was changed every 2 days. The sample was observed with a light sheet fluorescence microscope (Zeiss Light sheet Z.1) with a $5 \times$ NA 0.16 lens. Fluorescence was measured with excitation (488 $\mathrm{nm}$ ) and emission (SP 550 (Ch1) and LP585 (Ch2)) filters, and the GFP signal was obtained by subtracting $\mathrm{Ch} 2$ from Ch1. For image analysis, ZEN (Zeiss) and IMARIS 7.0 (Bitplane) were used.

\section{Additional files}

\begin{abstract}
Additional file 1: Movie S1. Active avoidance fear conditioning of wild type fish. The movie shows an example of the analysis. On day 1, wild type fish were placed in a white acrylic tank, and US (electric shock) was given $10 \mathrm{~s}$ after CS (green LED) was on. On day 5, the fish successfully escaped to another compartment after CS was on. (MOV 3374 kb)

Additional file 2: Figure S1. Performance of two-way active avoidance response of double transgenic (Gal4FF;UAS:ZBoTxBLC:GFP) fish. The following Gal4FF transgenic fish lines were crossed with

UAS:ZBOTxBLC:GFP effector fish, and analyzed for two-way active avoidance fear conditioning. a hspGGFF10C $(n=6)$, b hspGGFF20A $(n=10), \mathbf{c}$ hspGFF38B $(n=10)$, d hspGFF55B $(n=6)$, e SAGFF81B $(n=12), \mathbf{f}$ SAGFF226F $(n=9), \mathbf{g}$ SAGFF233A $(n=9)$, h SAGFF234A $(n=11), \mathbf{i}$ hspGFFDMC12A $(n=12), \mathbf{j}$ hspGFFDMC56B $(n=10), \mathbf{k}$ hspGGFF19B $(n=9)$, I hspGGFF19C $(n=9), \mathbf{m}$ hspGFF62A $(n=5), \mathbf{n}$ gata6SAGFF94A $(n=6)$, o SAGFF27C $(n=6), \mathbf{p ~ S A G F F 3 8 A ~}(n=8), \mathbf{q}$ SAGFF87C $(n=5), \mathbf{r}$ SAGFF92A $(n=8), \mathbf{s}$ SAGFF183A $(n=5), \mathbf{t}$ SAGFF195A $(n=6), \mathbf{u}$ SAGFF212C $(n=5), \mathbf{v}$ SAIGFF170B $(n=10)$, w hspGFFDMC76A $(n=10), \mathbf{x}$ hspGFFDMC85C $(n=11)$. Mean \pm SEM and avoidance $(\%)$ for individual fish are plotted. Performance of wild type fish $(n=28)$ described in Fig. 2 is shown in dotted lines. Two-way ANOVA, fish groups (wild type fish treated by CS-US, wild type fish treated by CS only and double transgenic fish including fish described in Figs. 2 and $3 \times$ training session days (day 1 , day 5 ), was performed $(F=7.236, P<0.0001$ ). Dunnett's multiple comparison post-hoc tests were performed between avoidance percentage of wild type fish and double transgenic fish on session day 1 and day 5. ${ }^{*} P<0.05$, ${ }^{* *} P<0.01,{ }^{* * *} P<0.001$, ${ }^{*}{ }^{* *} P<0.0001$; $\mathrm{ns}$, not significant $(P>0.05)$. a-j Reduced performance of the active avoidance response was observed. $k-x$ Performance of the active avoidance response was not significantly different between wild type and the double transgenic fish. (PPTX $8767 \mathrm{~kb}$ )
\end{abstract}

Additional file 3: Figure S2. GFP expression patterns of 16 Gal4FF;UAS:GFP fish that showed reduced performance of the active avoidance response. A dorsal view, a ventral view, and a schematic side view with positions of coronal sections are shown on the top. Serial coronal sections with position numbers are shown in the bottom. $\mathbf{a}$ hspGGFF10C, b hspGGFF20A, c hspGFF38B, d hspGFF55B, e SAGFF36B, f SAGFF70A, g SAGFF81B, h SAGFF120A, i SAGFF226F, j SAGFF228A, $\mathbf{k}$ SAGFF231A, I SAGFF233A, m SAGFF234A, n SAGFF234D, o hspGFFDMC12A, p hspGFFDMC56B. Scale bars in whole brain images: $500 \mu \mathrm{m}$. Scale bars in coronal section images: $200 \mu \mathrm{m}$. (PDF $3264 \mathrm{~kb}$ )

Additional file 4: Figure S3. GFP expression patterns in SAGFF120A;UAS:GFP fish at embryonic stages. Bright field and fluorescent images of frontal and lateral views of SAGFF120A;UAS:GFP fish at 24, 48, 72, and 96 hpf. Scale bar, 200 mm. (PPTX 1417 kb)

Additional file 5: Movie S2. Pavlovian fear conditioning of wild type fish. The movie shows an example of the analysis. Before conditioning: Wild type fish were placed in a white acrylic box and only CS (green LED) was given for $10 \mathrm{~s}$. The turning activity during CS was measured. During conditioning: US (electric shock) was given for $1 \mathrm{~s}, 9 \mathrm{~s}$ after CS was on. After conditioning: CS was given for $10 \mathrm{~s}$, and the turning activity was measured. (MOV $2802 \mathrm{~kb}$ )

Additional file 6: Movie S3. Alarm responses of wild type fish and SAGFF120A;UAS:ZBoTxBLCGFP fish. Behaviors of wild type and 
SAGFF120A:UAS:ZBoTxBLCGFP fish were videotaped upon addition of skin extract. (MOV $8664 \mathrm{~kb}$ )

Additional file 7: Movie S4. 3D image of the brain from SAGFF120A;UAS:GFP fish. A GFP fluorescence image of a transparent brain from SAGFF120A;UAS:GFP fish analyzed by light-sheet microscopy is shown. (MOV $6882 \mathrm{~kb}$ )

Additional file 8: Figure S4. GFP expression patterns in SAGFF120A;UAS:GFP and SAGFF120A;UAS:GFP;UAS:ZBOTXBLC:GFP fish. a Dorsal views of the brains from eight SAGFF120A;UAS:GFP ( 10 months old) fish and eight SAGFF120A;UAS:GFP;UAS:ZBoTxBLC:GFP ( 10 months old) fish are shown. Scale bars: $1 \mathrm{~mm}$. b Areas having more intensity than background (the maximum intensity measured in the posterior part of the telencephalon) were identified by using ImageJ [57] and shown in red. Scale bars: 500 um. c Immunohistochemistry using anti-GFP (green) and anti-NeuN (a neuronal marker, magenta) of coronal sections of the telencephalon and hypothalamus of brain samples from these transgenic fish. The fish numbers correspond to the numbers of the dorsal view

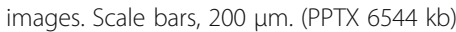

\section{Acknowledgments}

We thank N. Kishimoto and K. Sawamoto for instructions on brain dissection, H. Okamoto for advice on Pavlovian conditioning, and members of the Kawakami lab for fish maintenance and assistance in screening.

\section{Funding}

This work was partly supported by ERC Starting Grant 335561 (EY), National BioResource Project from Japan Agency for Medical Research and Development (AMED) (KK), and JSPS KAKENHI Grant Numbers JP15H02370, JP16H01651 and JP18H04988 (K.K.)

\section{Availability of data and materials}

All data generated during this study are included in this published article and its supplementary information files, and all materials generated during this study are available upon request.

\section{Authors' contributions}

$P L$ and KK designed the experiments and wrote the manuscript. PL, HT, DA YK, and EY performed experiments and analyzed the data. MLS generated the UAS:ZBOTxBLC:GFP transgenic line. MIt, Mlw, and HW performed anatomical analyses. AM assisted in the development of behavioral assay systems. All authors read and approved the final manuscript.

\section{Ethics approval}

This experiment was approved by the Institutional Animal Care and Use Committee (IACUC, approval identification number 27-2), and complied with the Guide for the Care and Use of Laboratory Animals of the IACUC.

\section{Competing interests}

The authors declare that they have no competing interests.

\section{Publisher's Note}

Springer Nature remains neutral with regard to jurisdictional claims in published maps and institutional affiliations.

\footnotetext{
Author details

'Division of Molecular and Developmental Biology, National Institute of Genetics, Mishima, Shizuoka 411-8540, Japan. ${ }^{2}$ Department of Genetics, SOKENDAI (The Graduate University for Advanced Studies), Mishima, Shizuoka 411-8540, Japan. ${ }^{3}$ Kavli Institute for Systems Neuroscience and Centre for the Biology of Memory, Norwegian Brain Centre, Norwegian University of Science and Technology (NTNU), Trondheim, Norway. ${ }^{4}$ Present address: Visual Interaction GmbH, Warthestrasse 21, 14513 Teltow, Germany. ${ }^{5}$ Present address: College of Liberal Arts and Sciences, Kitasato University, Sagamihara, Kanagawa 252-0373, Japan.
}

Received: 11 August 2017 Accepted: 7 March 2018

Published online: 25 April 2018

\section{References}

1. LeDoux JE. Emotion circuits in the brain. Annu Rev Neurosci. 2000;23:155-84.

2. Maren S. Neurobiology of Pavlovian fear conditioning. Annu Rev Neurosci. 2001;24:897-931.

3. Sah P, Faber ES, Lopez De Armentia M, Power J. The amygdaloid complex: anatomy and physiology. Physiol Rev. 2003;83(3):803-34.

4. Martinez-Garcia F, Novejarque A, Lanuza E. The evolution of the amygdala in vertebrates. In: Kaas JH, editor. Evolutionary Neuroscience. Cambridge, MA: Academic Press; 2009. p. 313-92.

5. Marek R, Strobel C, Bredy TW, Sah P. The amygdala and medial prefrontal cortex: partners in the fear circuit. J Physiol. 2013;591(10):2381-91.

6. Kapp BS, Frysinger RC, Gallagher M, Haselton JR. Amygdala central nucleus lesions: effect on heart rate conditioning in the rabbit. Physiol Behav. 1979; 23(6):1109-17.

7. LeDoux JE, Cicchetti P, Xagoraris A, Romanski LM. The lateral amygdaloid nucleus: sensory interface of the amygdala in fear conditioning. J Neurosci. 1990;10(4):1062-9.

8. Horner JL, Longo N, Bitterman ME. A shuttle box for fish and a control circuit of general applicability. Am J Psychol. 1961;74:114-20.

9. Pradel G, Schachner M, Schmidt R. Inhibition of memory consolidation by antibodies against cell adhesion molecules after active avoidance conditioning in zebrafish. J Neurobiol. 1999;39(2):197-206.

10. Eisenberg M, Kobilo T, Berman DE, Dudai Y. Stability of retrieved memory: inverse correlation with trace dominance. Science. 2003;301(5636):1102-4.

11. Amo R, Fredes F, Kinoshita M, Aoki R, Aizawa H, Agetsuma M, Aoki T, Shiraki T, Kakinuma H, Matsuda M, et al. The habenulo-raphe serotonergic circuit encodes an aversive expectation value essential for adaptive active avoidance of danger. Neuron. 2014;84(5):1034-48.

12. Nieuwenhuys R, Meek J. The telencephalon of actinopterygian fishes. In: Jones EG, Peters A, editors. Comparative Structure and Evolution of the Cerebral Cortex. New York: Springer; 1990. p. 31-73.

13. Wullimann MF, Mueller T. Teleostean and mammalian forebrains contrasted: evidence from genes to behavior. J Comp Neurol. 2004;475(2):143-62.

14. Mueller T, Guo S. The distribution of GAD67-mRNA in the adult zebrafish (teleost) forebrain reveals a prosomeric pattern and suggests previously unidentified homologies to tetrapods. J Comp Neurol. 2009;516(6):553-68.

15. Aoki T, Kinoshita M, Aoki R, Agetsuma M, Aizawa H, Yamazaki M, Takahoko M, Amo R, Arata A, Higashijima S, et al. Imaging of neural ensemble for the retrieval of a learned behavioral program. Neuron. 2013;78(5):881-94.

16. Braford MR Jr. Comparative aspects of forebrain organization in the rayfinned fishes: touchstones or not? Brain Behav Evol. 1995;46(4-5):259-74.

17. Northcutt RG. Connections of the lateral and medial divisions of the goldfish telencephalic pallium. J Comp Neurol. 2006;494(6):903-43.

18. Portavella M, Torres B, Salas C. Avoidance response in goldfish: emotional and temporal involvement of medial and lateral telencephalic pallium. J Neurosci. 2004;24(9):2335-42.

19. Lau BY, Mathur P, Gould GG, Guo S. Identification of a brain center whose activity discriminates a choice behavior in zebrafish. Proc Natl Acad Sci U S A. 2011;108(6):2581-6.

20. von Trotha JW, Vernier P, Bally-Cuif L. Emotions and motivated behavior converge on an amygdala-like structure in the zebrafish. Eur J Neurosci. 2014;40(9):3302-15.

21. Asakawa K, Suster ML, Mizusawa K, Nagayoshi S, Kotani T, Urasaki A, Kishimoto Y, Hibi M, Kawakami K. Genetic dissection of neural circuits by Tol2 transposon-mediated Gal4 gene and enhancer trapping in zebrafish. Proc Natl Acad Sci U S A. 2008;105(4):1255-60.

22. Kawakami K, Abe G, Asada T, Asakawa K, Fukuda R, Ito A, Lal P, Mouri N, Muto A, Suster ML, et al. zTrap: zebrafish gene trap and enhancer trap database. BMC Dev Biol. 2010;10:105.

23. Koide T, Miyasaka N, Morimoto K, Asakawa K, Urasaki A, Kawakami K, Yoshihara Y. Olfactory neural circuitry for attraction to amino acids revealed by transposon-mediated gene trap approach in zebrafish. Proc Natl Acad Sci U S A. 2009;106(24):9884-9.

24. Sternberg JR, Severi KE, Fidelin K, Gomez J, Ihara H, Alcheikh Y, Hubbard JM, Kawakami K, Suster M, Wyart C. Optimization of a neurotoxin to investigate the contribution of excitatory interneurons to speed modulation in vivo. Curr Biol. 2016;26(17):2319-28. 
25. Morita T, Nitta H, Kiyama Y, Mori H, Mishina M. Differential expression of two zebrafish emx homeoprotein mRNAs in the developing brain. Neurosci Lett. 1995;198(2):131-4.

26. Adolf B, Chapouton P, Lam CS, Topp S, Tannhauser B, Strahle U, Gotz M, Bally-Cuif L. Conserved and acquired features of adult neurogenesis in the zebrafish telencephalon. Dev Biol. 2006;295(1):278-93.

27. Ganz J, Kroehne V, Freudenreich D, Machate A, Geffarth M, Braasch I, Kaslin J, Brand M. Subdivisions of the adult zebrafish pallium based on molecular marker analysis. F1000Res. 2014;3:308.

28. Vazdarjanova A, Cahill L, McGaugh JL. Disrupting basolateral amygdala function impairs unconditioned freezing and avoidance in rats. Eur J Neurosci. 2001;14(4):709-18.

29. Jesuthasan SJ, Mathuru AS. The alarm response in zebrafish: innate fear in a vertebrate genetic model. J Neurogenet. 2008;22(3):211-28.

30. Speedie N, Gerlai R. Alarm substance induced behavioral responses in zebrafish (Danio rerio). Behav Brain Res. 2008;188(1):168-77.

31. Kroehne V, Freudenreich D, Hans S, Kaslin J, Brand M. Regeneration of the adult zebrafish brain from neurogenic radial glia-type progenitors. Development. 2011;138(22):4831-41.

32. Hama H, Kurokawa H, Kawano H, Ando R, Shimogori T, Noda H, Fukami K, Sakaue-Sawano A, Miyawaki A. Scale: a chemical approach for fluorescence imaging and reconstruction of transparent mouse brain. Nat Neurosci. 2011; 14(11):1481-8

33. Amorapanth $P$, LeDoux JE, Nader K. Different lateral amygdala outputs mediate reactions and actions elicited by a fear-arousing stimulus. Nat Neurosci. 2000;3(1):74-9.

34. Ribeiro AM, Barbosa FF, Munguba H, Costa MS, Cavalcante JS, Silva RH. Basolateral amygdala inactivation impairs learned (but not innate) fear response in rats. Neurobiol Learn Mem. 2011;95(4):433-40.

35. Ruhl T, Zeymer M, von der Emde G. Cannabinoid modulation of zebrafish fear learning and its functional analysis investigated by c-Fos expression. Pharmacol Biochem Behav. 2017;153:18-31.

36. Faustino Al, Tacao-Monteiro A, Oliveira RF. Mechanisms of social buffering of fear in zebrafish. Sci Rep. 2017;7:44329.

37. O'Connell LA, Hofmann HA. The vertebrate mesolimbic reward system and social behavior network: a comparative synthesis. J Comp Neurol. 2011; 519(18):3599-639.

38. Calandreau L, Jaffard R, Desmedt A. Dissociated roles for the lateral and medial septum in elemental and contextual fear conditioning. Learn Mem. 2007;14(6):422-9.

39. Herget U, Ryu S. Coexpression analysis of nine neuropeptides in the neurosecretory preoptic area of larval zebrafish. Front Neuroanat. 2015;9:2.

40. Viviani D, Charlet A, van den Burg E, Robinet C, Hurni N, Abatis M, Magara F, Stoop R. Oxytocin selectively gates fear responses through distinct outputs from the central amygdala. Science. 2011;333(6038):104-7.

41. Viktorin $G$, Chiuchitu C, Rissler M, Varga ZM, Westerfield M. Em×3 is required for the differentiation of dorsal telencephalic neurons. Dev Dyn. 2009;238(8):1984-98.

42. Simeone A, Gulisano M, Acampora D, Stornaiuolo A, Rambaldi M, Boncinelli E. Two vertebrate homeobox genes related to the Drosophila empty spiracles gene are expressed in the embryonic cerebral cortex. EMBO J. 1992;11(7):2541-50.

43. Gorski JA, Talley T, Qiu M, Puelles L, Rubenstein JL, Jones KR. Cortical excitatory neurons and glia, but not GABAergic neurons, are produced in the Emx1-expressing lineage. J Neurosci. 2002;22(15):6309-14.

44. Yoshida M, Suda Y, Matsuo I, Miyamoto N, Takeda N, Kuratani S, Aizawa S. Emx1 and Emx2 functions in development of dorsal telencephalon. Development. 1997;124(1):101-11.

45. Puelles L, Kuwana E, Puelles E, Bulfone A, Shimamura K, Keleher J, Smiga S, Rubenstein JL. Pallial and subpallial derivatives in the embryonic chick and mouse telencephalon, traced by the expression of the genes Dlx-2, Emx-1, Nkx-2.1, Pax-6, and Tbr-1. J Comp Neurol. 2000;424(3):409-38.

46. Brox A, Puelles L, Ferreiro B, Medina L. Expression of the genes Emx1, Tbr1, and Eomes (Tbr2) in the telencephalon of Xenopus laevis confirms the existence of a ventral pallial division in all tetrapods. J Comp Neurol. 2004;474(4):562-77.

47. Rink E, Wullimann MF. Some forebrain connections of the gustatory system in the goldfish Carassius auratus visualized by separate Dil application to the hypothalamic inferior lobe and the torus lateralis. J Comp Neurol. 1998; 394(2):152-70.

48. Villani L, Zironi I, Guarnieri T. Telencephalo-habenulo-interpeduncular connections in the goldfish: a Dil study. Brain Behav Evol. 1996;48(4):205-12.
49. Wall NR, De La Parra M, Callaway EM, Kreitzer AC. Differential innervation of direct- and indirect-pathway striatal projection neurons. Neuron. 2013;79(2): 347-60.

50. Asakawa K, Kawakami K. The Tol2-mediated Gal4-UAS method for gene and enhancer trapping in zebrafish. Methods. 2009;49(3):275-81.

51. Kawakami K, Asakawa K, Hibi M, Itoh M, Muto A, Wada H. Gal4 driver transgenic zebrafish: powerful tools to study developmental biology, organogenesis, and neuroscience. Adv Genet. 2016;95:65-87.

52. Goll MG, Anderson R, Stainier DY, Spradling AC, Halpern ME. Transcriptional silencing and reactivation in transgenic zebrafish. Genetics. 2009;182(3):747-55.

53. Urasaki A, Kawakami K. Analysis of genes and genome by the Tol2-mediated gene and enhancer trap methods. Methods Mol Biol. 2009;546:85-102.

54. Kurazono H, Mochida S, Binz T, Eisel U, Quanz M, Grebenstein O, Wernars K, Poulain B, Tauc L, Niemann H. Minimal essential domains specifying toxicity of the light chains of tetanus toxin and botulinum neurotoxin type A. J Biol Chem. 1992;267(21):14721-9.

55. Kishimoto N, Alfaro-Cervello C, Shimizu K, Asakawa K, Urasaki A, Nonaka S, Kawakami K, Garcia-Verdugo JM, Sawamoto K. Migration of neuronal precursors from the telencephalic ventricular zone into the olfactory bulb in adult zebrafish. J Comp Neurol. 2011;519(17):3549-65.

56. Burgess HA, Granato M. Modulation of locomotor activity in larval zebrafish during light adaptation. J Exp Biol. 2007;210(Pt 14):2526-39.

57. Image J Web Link. https://imagej.nih.gov/ij/. Accessed 20 Mar 2018.

58. Nagayoshi S, Hayashi E, Abe G, Osato N, Asakawa K, Urasaki A, Horikawa K, Ikeo K, Takeda H, Kawakami K. Insertional mutagenesis by the Tol2 transposonmediated enhancer trap approach generated mutations in two developmental genes: tcf7 and synembryn-like. Development. 2008;135(1):159-69.

59. Wullimann MF, Rupp B, Reichert H. Neuroanatomy of the Zebrafish Brain. Basel: Birkhäuser Verlag; 1996.

\section{Submit your next manuscript to BioMed Central and we will help you at every step:}

- We accept pre-submission inquiries

- Our selector tool helps you to find the most relevant journal

- We provide round the clock customer support

- Convenient online submission

- Thorough peer review

- Inclusion in PubMed and all major indexing services

- Maximum visibility for your research

Submit your manuscript at www.biomedcentral.com/submit
) Biomed Central 